\title{
Slow Pulse Repetition Interval Variation for High-Resolution Wide-Swath SAR Imaging
}

Felipe Queiroz de Almeida, Marwan Younis, Fellow, IEEE, Pau Prats-Iraola, Senior Member, IEEE, Marc Rodriguez-Cassola, Gerhard Krieger, Fellow, IEEE and Alberto Moreira, Fellow, IEEE

\begin{abstract}
In the context of spaceborne synthetic aperture radar (SAR) imaging, high resolution and wide swath are inherently conflicting requirements. These may however be simultaneously satisfied by advanced imaging modes with multichannel architectures in elevation and/or azimuth. The paper elaborates on a new mode based on multiple elevation beams and a simple PRI variation scheme which allows highresolution wide-swath imaging. It is shown to use the illumination time more efficiently than ScanSAR and yet to be simpler than staggered SAR. Good SAR imaging performance is achieved with a rather compact antenna design. The proposed imaging mode is suitable for spaceborne SAR systems with planar and reflector antennas. In order to improve the imaging performance, a reflector antenna architecture with a multichannel feed in both elevation and azimuth is considered.
\end{abstract}

Index Terms - Radar, Radar imaging, Spaceborne radar, Synthetic aperture radar, Digital beamforming, High resolution wide swath, SAR imaging modes

\section{INTRODUCTION}

$\mathrm{S}$ PACEBORNE Synthetic Aperture Radar (SAR) systems for remote sensing are subject to a well-known compromise between the best azimuth resolution and the maximum swath width [1]. Different single-channel SAR modes in fact mostly shift the emphasis towards either a high azimuth resolution or a wide swath. ScanSAR [2], [3], for instance, is a well-established imaging mode in which a wide swath composed of several subswaths is imaged by means of bursts alternately illuminating each sub-swath, as illustrated in Fig.1 (a). The system thus shares the available illumination time between a number of bursts covering different regions on the ground, trading-off azimuth resolution for a wider coverage. The alternating illumination introduces azimuth (Doppler) spectral gaps and limits the available bandwidth, but proper timing allows a given design azimuth resolution to be achieved. Moreover, mode parameters such as the pulse repetition frequency (PRF) can be set independently for each burst and thus tailored to optimize coverage and performance.

In contrast, multichannel architectures combined with digital beamforming (DBF) [4], [5], [6], [7], [8], [9], [10] show the capability of overcoming this limitation, in the sense of simultaneously delivering High-Resolution Wide-Swath (HRWS) SAR images and thus considerably out-performing single-channel systems.

Manuscript received April 20 ${ }^{\text {th }}$, 2020. F. Queiroz de Almeida, M. Younis, P. Prats-Iraola, M. Rodriguez-Cassola, G. Krieger and A. Moreira are with the Microwaves and Radar Institute of the German Aerospace Center (DLR), Oberpfaffenhofen, Germany (e-mail: felipe.queirozdealmeida@dlr.de).
One approach is to resort to a multichannel architecture in azimuth (Multi-Azimuth Channel, MAC) [11], to decouple the signal's azimuth sampling frequency from the PRF and thus increase the azimuth resolution without affecting the imaged swath. This method can also be used in conjunction with burst modes such as ScanSAR [12] to compensate for the burst operation-induced resolution loss, though the high squint variations may impact the performance.

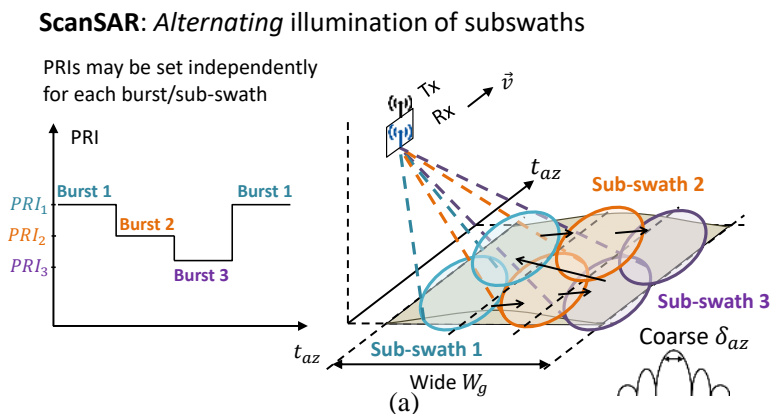

(a)

Multiple Elevation Beam (MEB) with constant PRI

Fixed Tx illumination, elevation beams correspond to different areas imaged simultaneously
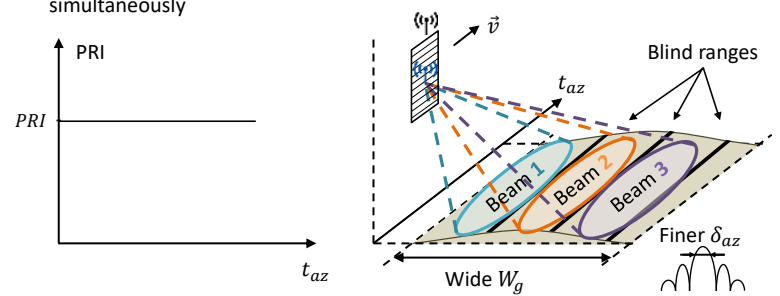

(b)

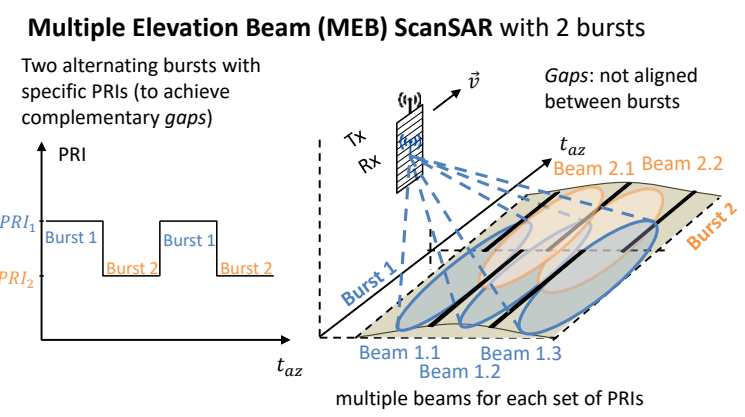

(c)

Fig.1: Schematic representation of discussed imaging modes. (a) Sub-swath variation in a conventional single-channel ScanSAR. (b) System with multiple elevation beams (MEB), by means of which simultaneously imaged sub-swaths separated by blind ranges are combined. The blind ranges are caused by transmission events and are azimuth invariant, due to the PRI regularity. (c) MEB system with two bursts with different PRIs, so that the blind ranges of one burst are covered by the other. 


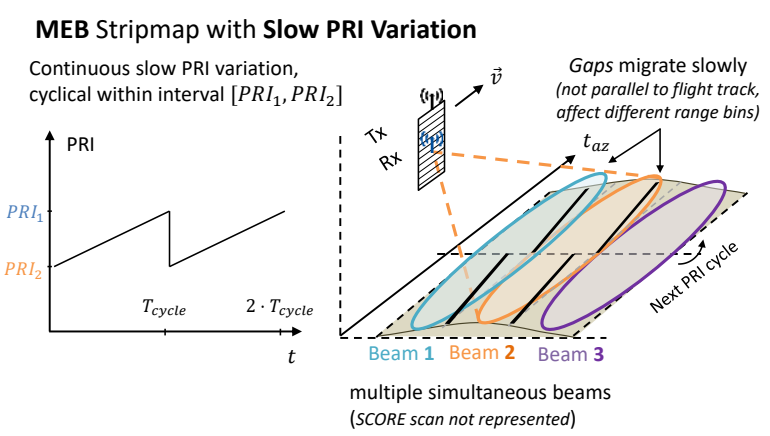

Fig.2: Schematic representation of proposed imaging mode: Multiple Elevation Beams (MEB) system with PRI variation from pulse to pulse, in which the gaps migrate continuously but slowly, affecting different range bins over azimuth. The spread of the gaps over multiple range bins (cf. Fig.4 (a) for an example gap diagram) causes Doppler spectrum gaps but not complete signal loss (blind range). Multiple simultaneous elevation beams are used (here the footprints are shown for a single pulse, scan is not represented) for ambiguity suppression.

Another family of methods derives from the use of multiple channels in elevation [7], [9], [10]. In fact, a system capable of simultaneously forming multiple elevation beams (MEB) through DBF can be used to image several sub-swaths at once. It represents an extension the SCan-On-Receive (SCORE) [13] concept, also known as SweepSAR [6], [14], [15].

As is the case in single-beam SCORE, typically a broad transmit (Tx) beam is used, to cover all the multiple sub-swaths, whereas simultaneous narrow receive $(\mathrm{Rx})$ beams are formed, following the echoes on ground. This is schematically represented in Fig.1 (b), for a constant-PRI Stripmap configuration, in which no spectral gaps in azimuth occur. Comparing this alternative with the MAC system architecture, the use of multiple channels in elevation often has the advantage of leading to a more compact antenna design [9]. This is due to the fact that the typical SAR antenna requires a broader beam in elevation than in azimuth and is thus larger in the latter dimension, which is generally also true for each aperture of a multichannel antenna system. In the latter case, the azimuth reconstruction performance is in addition sensitive to the spacing of the channels, which often leads to large antenna dimensions, especially for lower PRFs. An additional advantage of multichannel architectures in elevation is simpler signal processing, making on-board processing more feasible with current technology.

Both the MAC and MEB system architectures are, however, subject to an inherent limitation in the form of blind ranges between the sub-swaths. These occur because it is not possible to record the echoes while transmitting, a characteristic of monostatic systems which leads to gaps in the recorded echo. The gaps have regularly-spaced positions determined by the (constant) PRF. As mentioned, ScanSAR allows different PRFs to be used for each burst, and thus an interesting extension of these systems [7], [9] would be a multi-elevation beam ScanSAR with two-bursts, in order to cover the blind ranges of one burst with the other, as illustrated in Fig.1 (c). In this case, the pulse repetition interval (PRI) has two values, one for each burst, chosen as to be complementary in terms of the blind ranges. The instantaneous PRI curve may thus be seen as a square-wave, which can also be interpreted as a particularly simple form of PRI staggering (understood in a general sense as PRI variation), analyzed in a broader context in [16]. The burst operation has, however, the disadvantage of azimuth spectral gaps introduced by the alternating illumination, determined by the burst lengths.

The new strategy originally suggested in [7] and analyzed here in more detail is to allow the PRI to slowly vary between the two extreme values, as illustrated in Fig.2. A slow and linear PRI variation between two extreme values (repeating cyclically) is proposed. The PRI curve thus becomes a saw-tooth waveform. As a consequence, the blind ranges migrate slowly across the swath, according to the instantaneous PRI. Spectral gaps due to the azimuth illumination will still occur, but in this case their length will be determined by the extent of the blind range region, which is linked to the pulse duty cycle, instead of the burst duration. This means that an opportunity arises to increase the observation time in comparison to a conventional ScanSAR mode. The PRI variation has the downside of leading to a nonuniformly sampled azimuth signal, which may be nonetheless adequately recovered by interpolation, at the cost of oversampling in azimuth. Moreover, in this case, the slow variation leads to relatively small deviations from uniform sampling, and linear interpolation may be used instead of more advanced algorithms (such as [16]). This method may be referred to as the Slow PRI Variation with Multiple Elevation Beams (SPV-MEB) method, and will be analyzed in Section II in detail. Note that the strategy differs from the fast PRI variation described in [16], [17] which interpolates across the gaps and requires more sophisticated processing and higher oversampling on average.

A mathematical description of the mode and design considerations are provided in Section II, followed by considerations of mode variations and other mission aspects in Section III. Then, Section IV provides performance simulations examples for the aforementioned mode. Finally, the paper is concluded in Section V with a summary and discussion of the introduced concepts and the performance assessment results.

\section{Mode Description AND Design Considerations}

This section describes the aforementioned slowly varying PRI mode mathematically and discusses its properties. Section II.A addresses the timing characteristics of the mode and derives a criterion for a first-order design of the parameters. Section 0 discusses the signal's Doppler spectrum.

\section{A. Timing Analysis and Sequence Design}

To analyze this mode in more detail, we consider, first of all, a constant PRF SAR operated at a regular pulse repetition interval of PRI seconds between pulses. A monostatic system is considered, subject to gaps in the receive signal due to transmission events. Timing constraints due to Nadir returns are not considered, as these are assumed to be mitigated by proper design of the antenna patterns (cf. [18] for a useful elevation beamforming technique and the impact of Nadir returns in a multiple elevation beam staggered SAR system). 
Let the return order, or equivalently the number of travelling pulses (i.e., the number of pulses transmitted before the echo of any given pulse returns), be defined as

$k=\left\lfloor\frac{2 \cdot R_{0}}{c} \cdot \frac{1}{P R I}\right\rfloor$

where $R_{0}$ is a given slant range and $c$ is the speed of light. The floor operation \ 」 is taken, as only integer values of $k$ have a physical interpretation (a non-integer value means that the return of the $(k+1)^{t h}$ pulse did not yet occur for that given range). In this case, pulse transmission events occur at regular intervals PRI seconds apart, with duration $\tau_{P}$. The corresponding blind ranges [19], where signal echoes cannot be recorded due to the pulse transmission, are in this case given by

$$
\frac{c}{2} \cdot k \cdot P R I \leq R_{\text {blind }}(k) \leq \frac{c}{2} \cdot k \cdot P R I+\tau_{P}
$$

Typical values of $k$ are in the order of 10 to 30 .

Now, we allow the system's PRI to undergo a slow linear variation between $P R I_{\text {min }}$ and $P R I_{\text {max }}$, during a period of $T_{\text {cycle }}$ seconds. If the swath of interest extends between $R_{\min }$ and $R_{\text {max }}$, the return orders of interest lie in the interval

$$
\left\lfloor\frac{2 \cdot R_{\min }}{c} \cdot \frac{1}{P R I_{\max }}\right\rfloor \leq k \leq\left\lfloor\frac{2 \cdot R_{\max }}{c} \cdot \frac{1}{P R I_{\min }}\right\rfloor
$$

We first assume for simplicity that the PRI variation sequence is long and smooth enough to be treated as continuous, and that the effect of the PRI difference during the pulse travelling time ${ }^{1}$ can be neglected at this point. Thus, one may write:

$$
P R I\left(t_{a z}\right)=P R I_{\max }-\frac{\Delta P R I}{T_{\text {cycle }}} \cdot\left(t_{a z} \bmod T_{\text {cycle }}\right),
$$

where $\triangle P R I=P R I_{\max }-P R I_{\min }$ and $t_{a z}$ denotes slow time (i.e. azimuth time). The ranges corresponding to the beginning and end of the blocked return regions are then given, for a fixed order $k$, by

$$
\begin{aligned}
& R_{1}\left(t_{a z}\right)=\frac{c}{2} \cdot k \cdot\left(P R I_{\text {max }}-\frac{\Delta P R I}{T_{\text {cycle }}} \cdot t_{a z}\right), \\
& R_{2}\left(t_{a z}\right)=\frac{c}{2} \cdot k \cdot\left(P R I_{\text {max }}-\frac{\Delta P R I}{T_{c y c l e}} \cdot t_{a z}\right)+\tau_{P} ;
\end{aligned}
$$

respectively (compare to (2)), as represented schematically in Fig.3.

Hence, under the nearly constant PRI approximation, the azimuth gap length $T_{\text {gap }}$ for a given range $R_{0}$ can be obtained by setting $R_{0}=R_{1}\left(t_{1}\right)=R\left(t_{2}\right)$ in (5) and calculating $T_{\text {gap }}=t_{1}-t_{2}$, which leads to

$$
T_{\text {gap }}(k)=\frac{\tau_{P}}{k} \cdot \frac{T_{\text {cycle }}}{P R I_{\max }-P R I_{\min }} .
$$

\footnotetext{
${ }^{1}$ In reality, the round-trip delay makes the time in which a pulse is transmitted dependent on the PRI which was adopted a number of pulses before. This is considered in e.g. (9) but the simplified model given here is adopted first to find a firstorder estimation of the involved parameters and ease understanding.
}

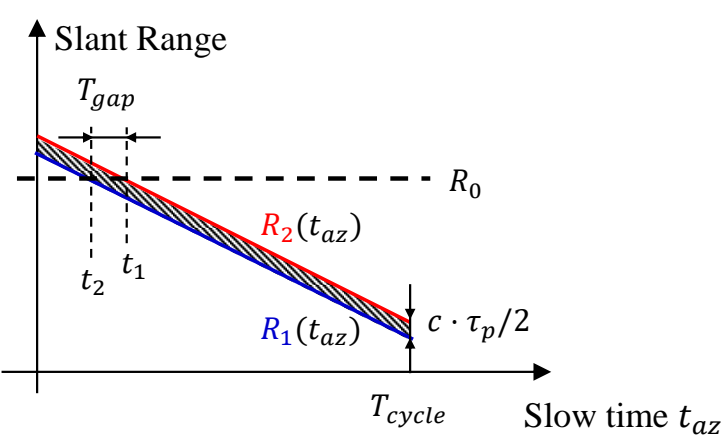

Fig.3: Schematic representation of blind ranges. $R_{1}\left(t_{a z}\right)$ and $R_{2}\left(t_{a z}\right)$ show the beginning and end of the gaps against slow time. The rate of migration of the gaps in range (ordinates of the plot) - caused by the transmit events determines the duration of the gaps in azimuth (abscissa of the plot). At a given slant range $R_{0}, t_{2}$ and $t_{1}$ represent the times in which $R_{2}\left(t_{2}\right)=R_{0}$ and $R_{1}\left(t_{1}\right)=R_{0}$, that is, the instants at which the range of interest enters and leaves the azimuth gap. The duration of the gap in slow time is then $T_{\text {gap }}=t_{1}-t_{2}$.

It is interesting to note that the gap length is implicitly a function of the range since the order parameter $k$ varies with range. Moreover, larger gaps tend to occur at near range, for a given PRI value, and the gap length for a given range is inversely proportional to the extent of PRI variation. As expected, the limit case of no PRI variation leads to an arbitrarily long gap, meaning the range in question is effectively a blind range.

\section{1) Extent of Blockage Region}

The extent of the PRI variation $\triangle P R I=P R I_{\max }-P R I_{\min }$ is a key parameter for the design of the mode and is analyzed in the following. It should be noted that, albeit long, the PRI variation sequence is discrete. We thus drop the earlier assumption of a continuous PRI variation and assume a sequence of length $N_{P R I}$ so that the PRI varies as

$P R I[n]=P R I_{\max }-n \cdot \frac{\Delta P R I}{N_{P R I}-1}, \quad 0 \leq n \leq N_{P R I}-1$,

leading to a cycle of length

$$
T_{\text {cycle }}=\sum_{n=0}^{N_{P R I}-1} P R I[n]=N_{P R I} \cdot\left(P R I_{\max }-\frac{\Delta P R I}{2}\right)
$$

where, as a consequence of the slow variation assumption, $T_{\text {cycle }} \gg \triangle P R I$ while $N_{P R I}$ is typically in the order of a few thousands up to tens of thousands. The time interval it takes to transmit $k$ pulses is:

$$
d_{i, k}=\sum_{n=i}^{i+k-1} P R I[n]
$$

which corresponds to the beginning of the $k^{\text {th }}$ order blockage event starting from pulse $i$. Substituting (7) into (9) leads to

$$
\begin{array}{lc}
d_{i, k}=k \cdot P R I_{\text {max }}-\frac{\Delta P R I}{2 \cdot\left(N_{P R I}-1\right)} \cdot(2 \cdot i+k-1) \cdot k, \\
\text { for } 0 \leq i \leq N_{P R I}-k & (\text { region } I) ;
\end{array}
$$


$d_{i, k}=T_{\text {cycle }}-\left(N_{P R I}-k\right) \cdot P R I_{\max }$

$+\frac{\Delta P R I}{2 \cdot\left(N_{P R I}-1\right)} \cdot\left(2 \cdot i+k-N_{P R I}-1\right) \cdot\left(N_{P R I}-k\right)$,

for $N_{P R I}+1-k \leq i \leq N_{P R I}-1 \quad$ (region $I I$ ).

For a given $k$, the range boundaries of the blockage region are given by $R_{1}[i]=c / 2 \cdot d_{i, k}$ and $R_{2}[i]=c / 2 \cdot d_{i, k}+\tau_{P}$, respectively (compare to (5)). The extent of each of the blockage regions may be obtained by taking the maximum and minimum of (10) over the indices $i$ for a fixed $k$ and converting to range. This leads to the limits:

$$
\begin{aligned}
& R_{\min }^{b}(k)= \\
& \quad \frac{c}{2} \cdot\left(k \cdot P R I_{\text {max }}-\frac{\Delta P R I}{2 \cdot\left(N_{P R I}-1\right)} \cdot\left(N_{P R I}-k-1\right) \cdot k\right) \\
& R_{\max }^{b}(k)= \\
& \frac{c}{2} \cdot\left(k \cdot P R I_{\text {max }}-\frac{\Delta P R I}{2 \cdot\left(N_{P R I}-1\right)} \cdot(k-1) \cdot k+\tau_{P}\right)
\end{aligned}
$$

\section{2) Criteria for PRI Variation Design}

A meaningful design criterion for the PRI variation is to ensure that the blockage regions of different orders $k$ do not overlap, in the sense that a given range within the imaged swath does not belong to more than one region. The violation of this condition causes two large gaps to occur, impairing azimuth performance. In other words, it should be ensured that

$$
R_{\min }^{b}(k)>R_{\max }^{b}(k-1)
$$

for all $k \mathrm{~s}$ of interest within the swath. Substituting (11) into (12) leads to the condition

$P R I_{\text {max }}-\frac{\Delta P R I}{N_{P R I}-1} \cdot\left(\left(N_{P R I}-k-1\right) \cdot k+1\right)-\tau_{P}>0$,

which can be further simplified taking into account that the sequence is very long, thus $N_{P R I}-k-1 \cong N_{P R I}$ and $N_{P R I}-1 \cong N_{P R I}$ as $N_{P R I} \gg k$. The simplified condition is hence

$$
P R I_{\max }-\triangle P R I \cdot(k)-\tau_{P}>0 .
$$

As $P R I_{\max }$ is usually determined from the azimuth sampling requirements, it remains to determine the maximum PRI variation. Substituting the maximum return order $k_{\max }$ of interest within the swath as given by (3) into (14) yields the approximate critical (maximum) PRI variation

$$
\Delta P R I_{\max } \cong \frac{P R I_{\max }-\tau_{P}}{k_{\max }} .
$$

Substituting the above into (6) yields the azimuth gap length (duration) for a given order $k$ as

$$
\begin{gathered}
T_{\text {gap }}(k)=T_{\text {cycle }} \cdot \frac{k_{\text {max }}}{k} \cdot \frac{\tau_{P}}{P R I_{\max }-\tau_{P}} \\
T_{\text {gap }}(k)=T_{\text {cycle }} \cdot \frac{k_{\text {max }}}{k} \cdot \frac{d c_{\text {min }}}{1-d c_{\min }}
\end{gathered}
$$

where $d c_{\min }$ is the (minimum) pulse duty cycle ${ }^{2}$ and the substitution $\tau_{P}=d c_{\min } \cdot P R I_{\max }$ was used.

3) Cycle Time Design

As the critical illumination time is at far range, $T_{i l l}=\lambda \cdot R_{\max } /\left(2 \cdot v \cdot \delta_{a z}\right)$, i.e., for $k=k_{\max }$, the cycle time should satisfy

$$
\begin{gathered}
T_{\text {cycle }}=T_{\text {ill }}+T_{\text {gap }}\left(k_{\text {max }}\right) \\
T_{\text {cycle }}=\frac{\lambda \cdot R_{\max }}{2 \cdot v \cdot \delta_{A Z}} \cdot \frac{1-d c_{\min }}{1-2 \cdot d c_{\min }},
\end{gathered}
$$

where $\lambda, v$ and $\delta_{A Z}$ denote the wavelength, platform velocity and required azimuth resolution, respectively. The equation above gives a criterion for the design of the cycle time, provided the PRI variation is sufficient to prevent overlap in range from neighboring blockage regions (i.e. (15) is satisfied).

This result also indicates a -counter-intuitive- "compression" of the gap duration for larger ranges (higher $k$ ). This is advantageous, as it allows increasing the illumination time $T_{i l l}$ with range, which is needed to provide a constant Doppler bandwidth (compensating the decrease in Doppler rate with increasing range [1]).

\section{4) Comparison to ScanSAR}

Note that the duration of the azimuth gap translates into the extension of the Doppler spectral gap. Here, the gap duration is a small fraction of the cycle time $T_{\text {cycle }}$ which depends upon the pulse duty cycle. Typical values for the gap extent (cf. (16) and Section IV), are in the order of $8-16 \%$ of the cycle. This is in contrast to the ScanSAR mode: for $N_{s}$ sub-swathes, the mean gap-to-cycle-time ratio is approximately [20], [21]

$$
\frac{\bar{T}_{\text {gap }}}{T_{\text {cycle }}}=\frac{N_{s}-1}{N_{s}}
$$

which is in the range of $[0.5,1)$. This means that as a rule shorter gaps are expected in the slow PRI variation mode than in ScanSAR. This implies that the cycle time is used more efficiently (in the sense of a lower proportion of gaps in the cycle), and moreover an increased control over the maximum squint angle (a consequence of the Doppler gap length) is made possible, as $d c_{\text {min }}$ may be set more freely than the (integer) number $N_{S}$.

\section{5) PRI Sequence Design Example}

In practice, a simple approach to design the sequence design is to take $P R I_{\max }$ as a parameter and apply (15) together with (3) to iteratively estimate $P R I_{\min }, k_{\min }$ and $k_{\max }$. Knowledge of $P R I_{\text {min }}, P R I_{\text {max }}$ and $T_{\text {cycle }}$ from (17) defines the sequence. If a fixed average PRI is desired, the initial choice of $P R I_{\max }$ can be revised and the procedure repeated iteratively until a sequence with the desired properties is found.

\footnotetext{
${ }^{2}$ The pulse duration $\tau_{p}$ is assumed to be fixed, regardless of the PRI variation. Therefore, the pulse duty cycle changes with time, with minimum value $d c_{\text {min }}=\tau_{P} / P R I_{\text {max }}$.
} 
This strategy was used to design a PRI sequence suitable for imaging a $400 \mathrm{~km}$ swath corresponding to $18<k<25$. Here the PRF varies between $3300 \mathrm{~Hz}^{3}$ and $3433 \mathrm{~Hz}$ every $T_{\text {cycle }}=3.52$ seconds. The abscissa values show a total of 3 cycles. Note that the full set of parameters is given in Section IV.A which provides a complete system design example (cf. TABLE II.). The timing is described through the blockage diagram, shown in Fig.4 (a), which visualizes the migration of the blocked ground ranges (red stripes) versus azimuth time.

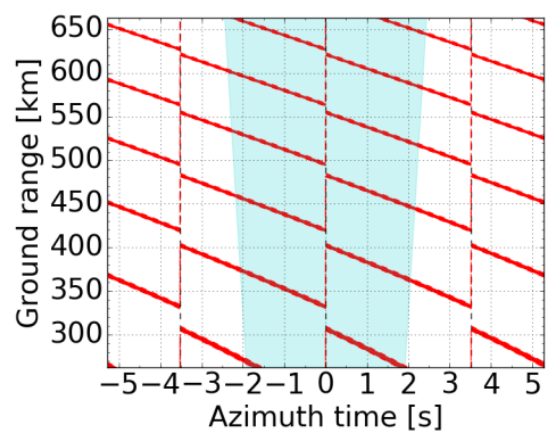

(a)

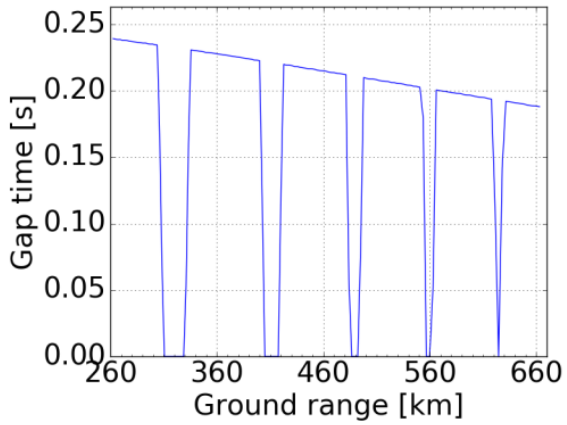

(b)

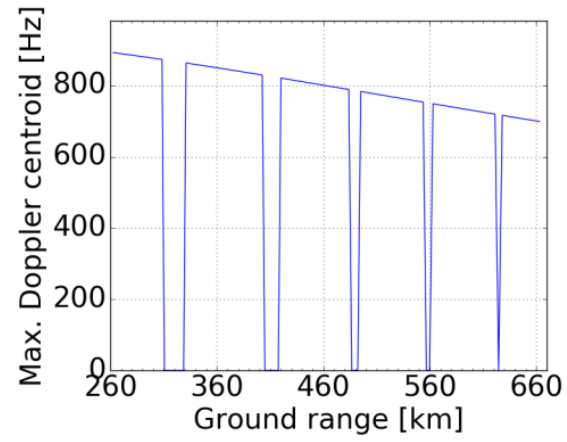

(c)

Fig.4: Illustration of timing considerations for the slowly varying PRI concept. (a) Blockage diagram with all orders $18 \leq k \leq 25$ for a $400 \mathrm{~km}$ swath, following the parameters of TABLE I and TABLE II (mean PRF of $3467.15 \mathrm{~Hz}$ ). The blue region highlights the antenna beamwidth in azimuth. (b) Duration of the main gap as a function of ground range, for the same system. It is smaller than $0.24 \mathrm{~s}$, and better in far range. (c) Worst-case Doppler centroid imposed by the gaps: the maximum value is $892 \mathrm{~Hz}$, in near range.

${ }^{3}$ For this example, $P R I_{\max }$ was fixed to match this parameter, taken as in input.
For each order $k$, a pattern resembling Fig. 3 is shown. The vertical "jumps" are due to sudden PRI changes at the end of the cycle time, though their blockage contribution is irrelevant. For each range, an imaginary horizontal line whose length equals the illumination time $\left(T_{i l l}=3.44 \mathrm{~s}\right.$ in far range) is intercepted only once by the blockage, which shows that the design is valid. The illumination-time line is, in general, not centered around zero azimuth time, thus indicating the processed Doppler bandwidth is not centered at $0 \mathrm{~Hz}$. The light blue shaded area marks the extreme shifts of the illumination line necessary to achieve the required resolution.

Fig.4 (b) shows the duration of the main gap at each ground range, and reveals that certain ranges without gaps exist, separating the 6 regions of blockage. A corresponding number of elevation beams is required to cover the swath. The distance between the last two regions is seen to be small, but no overlap occurs, indicating that the criterion established in (12) is fulfilled by the design.

The timing considerations in this section do not take into account the fact that, in practice, the radar system's PRI values are often quantized (e.g. to an integer multiple of the A/D converter's sampling interval). This imposes a maximum difference between two consecutive PRI values (expected to be in the order of 5-10 ns with current technology) which may be violated by following the design criteria shown here (e.g. in Section IV the design examples lead to changes in the order of $1 \mathrm{~ns}$ between consecutive PRIs). Though simulation results to this topic are not shown for the sake of brevity, the PRI sequences following the criteria described here may be adapted by keeping the PRI constant for e.g. 5-10 pulses (according to the minimum possible change) and then applying the minimum allowed jump, while keeping the same overall PRI span during the cycle time. This results in a coarser "staircase" waveform approximating the PRI ramp with negligible impact on the timing (as the relevant delays are "smoothed" by the order $k$ ) and small impact (typically less than a dB worsening of the azimuth ambiguity level for the same average PRF) on the resampling/azimuth performance.

\section{B. Azimuth Spectrum}

\section{1) Spectral Gap Position}

In the case of the traditional ScanSAR mode [20], a finite number of well-defined bursts of pulses occur, each related to a given sub-swath. For a given range (belonging to a specific sub-swath), the time in which the system is illuminating the other swath(s) translates into a gap of duration $T_{g a p}$ over which no data are gathered over the synthetic aperture. The lack of data translates into a (Doppler) spectral gap. In contrast, the remaining time of the cycle $T_{i l l}=T_{\text {cycle }}-T_{\text {gap }}$ provides the illumination of the target, and has to be long enough so that the needed bandwidth for the particular resolution is acquired. The Doppler centroid of the signal in azimuth is determined by the target's position with respect to the swath's illumination cycle, causing an azimuth-variant performance. 
In the slow PRI variation mode, the burst (here understood as a period of continuous illumination) is interleaved with spectral gaps, as illustrated in Fig.5 (a).

They are nonetheless shorter, and dictated by the pulse duty cycle, following (16). In the figure, the best and worst cases of Doppler centroid are highlighted, corresponding to the zeroDoppler times $t_{0}^{b}$ and $t_{0}^{w}$, respectively. All targets within a particular range fall in-between these two extreme cases. The focusing requires for full resolution only a single burst at a time (e.g., the one closest to a given zero Doppler time), since by design the usable part of the spectrum allows at least one continuous observation interval of duration $T_{i l l}$ which is long enough to achieve the resolution at all ranges. The extra illumination time, however, can be exploited to yield additional looks (cf. Section II.B.3) or alternatively reduced by additional azimuth beamforming (cf. III.B).

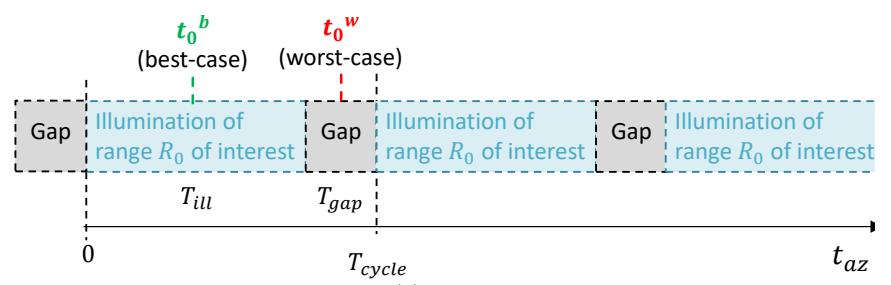

(a)

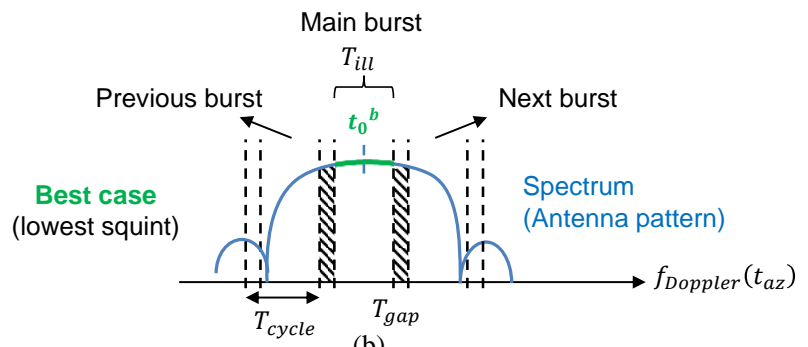

(b)

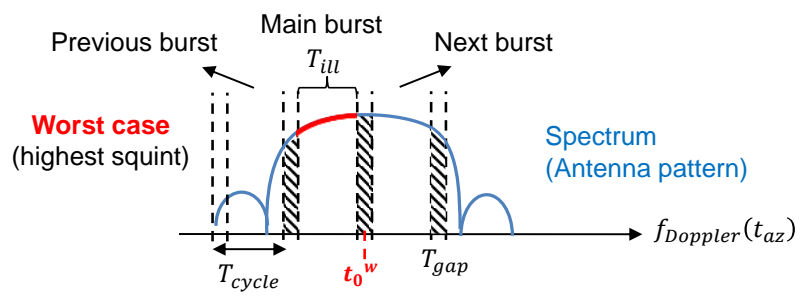

(c)

Fig.5: Schematic representation of spectral gaps induced by "burst"-like operation. (a) Cycle time divided, for a particular range, into the observation time $T_{i l l}$ and gap time $T_{\text {gap }}$. The zero Doppler times $t_{0}^{b}$ and $t_{0}^{w}$ lead to the best and worst cases, respectively, in terms of Doppler centroid. (b) Best case (no Doppler centroid): zero Doppler time $t_{0}^{b}$ in the center of the illumination, the spectrum lies in the antenna's main beam. (c) Worst case (highest Doppler centroid): zero Doppler time $t_{0}^{w}$ in the center of the gap, causing a gain loss in the main beam and imposing the highest Doppler centroid on the data.

\section{2) Time-Frequency Diagrams and First-Order Processing Aspects}

The properties of the spectrum are illustrated in Fig. 6 by means of time-(Doppler) frequency diagrams. Fig.6 (a) shows the "raw data domain", i.e., before any processing. Individual targets at minimum slant range $R_{0}$ are represented as lines of inclination given by the Doppler rate $\xi_{\text {Dop }}=-2 \cdot v^{2} /\left(\lambda \cdot R_{0}\right)$ [1]. It is apparent how the timing of the gaps translates into spectral gaps, which depend on the position of the target in azimuth. Both the "best case" and the "worst case" discussed before are shown. The second diagram (cf. Fig.6 (b)) considers processing in the sense that each target is registered to its zero Doppler crossing time $\left(t_{0}^{b}\right.$ and $t_{0}^{w}$ for the best and worst case, respectively). Note that the center of the spectrum is taken for the best case, but a linearly varying Doppler centroid is imposed on the data by the gaps (inclination $-\xi_{\text {Dop }}$ ).

The configuration is similar to a ScanSAR signal [22], except for the duration of the gaps. An analogous processing strategy would thus be applicable. An option in order to extract the different looks could be to apply a burst-wise (cycle-wise) de-rotation [22] by multiplication of the signal with a complex exponential of the form

$$
\phi_{d e-r o t}(t)=\exp \left(-j \cdot \pi \cdot \xi_{D o p} \cdot\left(t-t_{r e f}\right)^{2}\right) .
$$

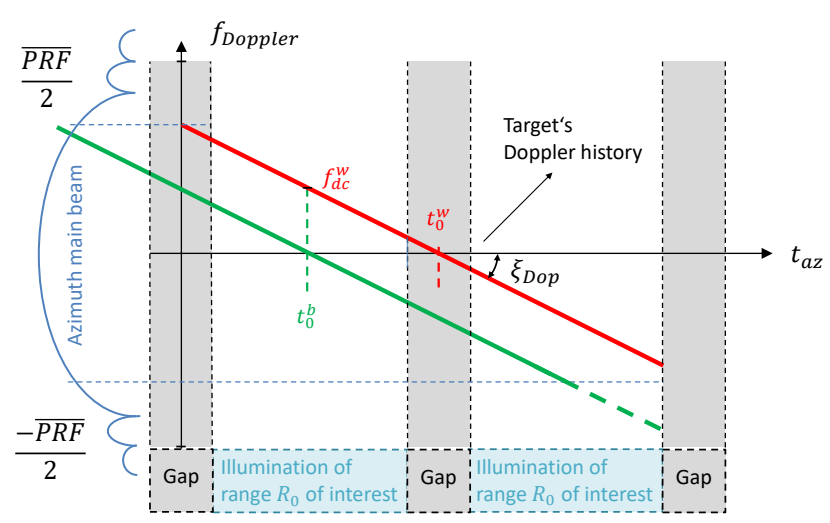

(a)

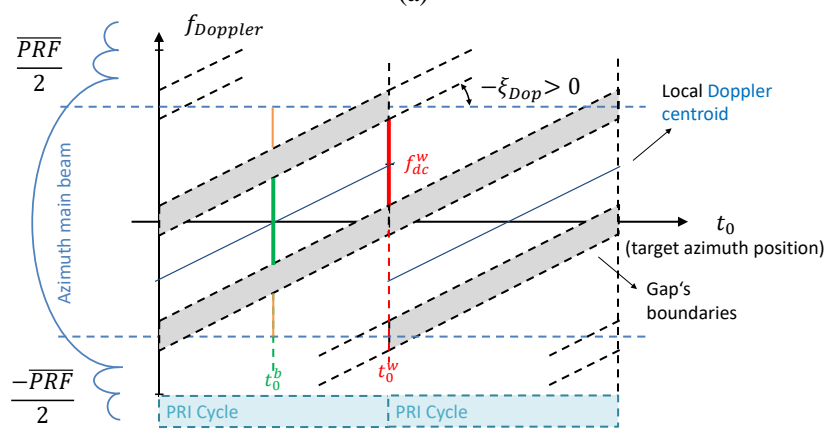

(b)

Fig.6: Time-frequency diagrams (Doppler) for targets imaged in the slow PRI mode, assuming stationary targets at a fixed minimum slant range. (a) Diagram in "raw data domain", i.e., without any processing. (b) Diagram in "image domain", assuming registering of the targets to their zero Doppler crossing time.

Such a processing step, possibly in combination with a sampling rate expansion, could "align" the gaps and allow the use of conventional filtering for the extraction of the looks as might be required, e.g., to generate a multilooked image or for interferometry (cf. Section III.D.3).

It is also apparent from the representation, as will be further discussed next, that excess bandwidth is acquired: in the worst case (i.e. highest squint) position, another look with the same bandwidth could be formed by considering the negative Doppler portion of the spectrum. 


\section{3) Doppler Diversity and Multilook Potential}

The azimuth spectrum analysis in the previous sub-sections shows that the required beamwidth of the azimuth patterns lead to illumination of a Doppler bandwidth in excess of what is required to achieve the resolution $\delta_{A Z}$, regardless of the position of the Doppler gaps. Each target is seen from at least one, but typically two, further bursts (cf. Fig.5 (b) and (c)), but with a duration potentially shorter than $T_{i l l}$. This additional and free information could be used, e.g., for interferometric multilooking or estimates exploiting squint angle diversity, as will be detailed next.

Fig.7 (a) illustrates the timing of the worst-case target position, with a gap at zero Doppler. The corresponding (non-continuous) observation time is $T_{\text {beam }}=2 \cdot\left(T_{i l l}+T_{\text {gap }}\right)$, and the useful part of the spectrum lies within $\left[-T_{\text {cycle }}, T_{\text {cycle }}\right]$ from the zero-Doppler time. In Fig.7 (b), a generic target position is considered, and the corresponding gap starts at a time $t_{g}$. Continuous observation with duration $T_{i l l}$ is guaranteed by design, but the additional observation time is split into two unequal intervals, of durations $\Delta t_{1}$ and $\Delta t_{2}$. The worst case seen before corresponds to $t_{g}=0, \Delta t_{1}=0$ and $\Delta t_{2}=T_{i l l}$. The other extreme is the best case in Fig.7 (c), with $t_{g}=-T_{\text {cycle }} / 2$ and $\Delta t_{1}=\Delta t_{2}=T_{i l l} / 2$. In general, $\Delta t_{1}+$ $\Delta t_{2}=T_{i l l}$, meaning that a continuous observation time of at least $T_{i l l} / 2$ is always available.

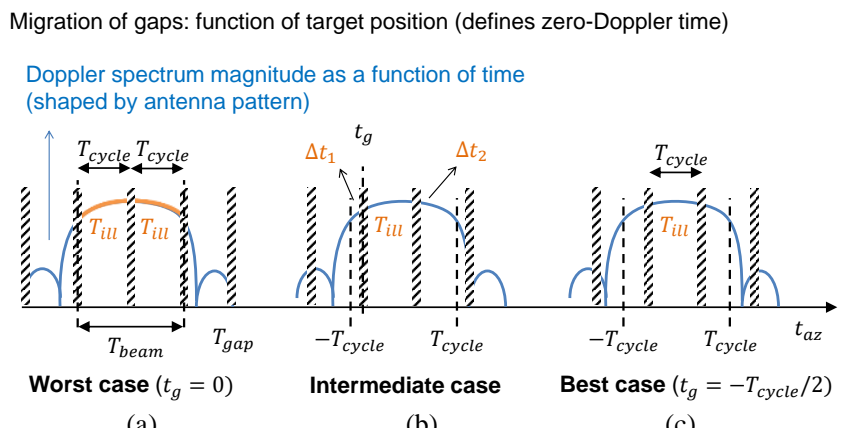

(a)

(b)

(c)

Fig.7: Spectral gaps changing according to target position, as an illustration of the excess Doppler bandwidth acquired and the multilooking potential. (a) Worst case (gap at zero Doppler). (b) Intermediate (generic) case. (c) Best case (cycle centered around zero Doppler).

A trade-off between azimuth resolution and (non-thermal) noise rejection ${ }^{4}$ could thus be introduced, which may be exploited to suit the needs of different applications. If the resolution goal $\delta_{A Z}$ used for the design of $T_{i l l}$ (cf. (17)) is relaxed by an even (for convenience) integer factor $N_{\text {res }} \geq 2$, this means that at least $N_{l}=3 \cdot N_{\text {res }} / 2$ (nominal) looks can be formed from the same data. Clearly, the effective number of looks achieved depends on the shape of the azimuth patterns, since not all the looks have the same power. The effective number of looks is thus smaller than the nominal one, increasingly approaching it

\footnotetext{
${ }^{4}$ Recall that speckle is a non-thermal noise source which ensues from the large number of coherent scatterers in a single resolution cell. Though a signal-to-noise ratio (SNR) with respect to both thermal and non-thermal noise may be defined for multilook images [1], incoherent de-speckling is considered not to change the signal-to-thermal noise ratio or the NoiseEquivalent Sigma Zero (NESZ) according to the definitions assumed in this paper.
}

for flatter patterns. Nonetheless, the factor of improvement in the noise variance may be higher than the factor of resolution degradation, which can be of interest for several applications, e.g. interferometry (cf. [23] for a thorough discussion of the tradeoffs involved in along-track interferometric performance, including the potential benefits of multilooking). For example, using the parameters of TABLE II and taking the exemplary case of $N_{\text {res }}=5$, an azimuth resolution of $25 \mathrm{~m}$ could still be achieved with 10-11 nominal looks. Next, the effective number of looks is computed using two example patterns shown in Fig.8.

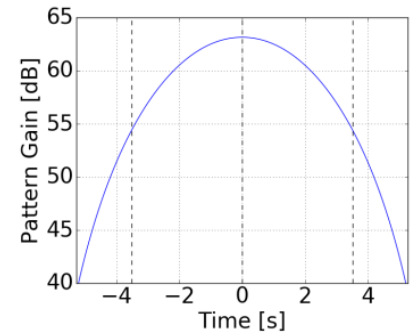

(a)

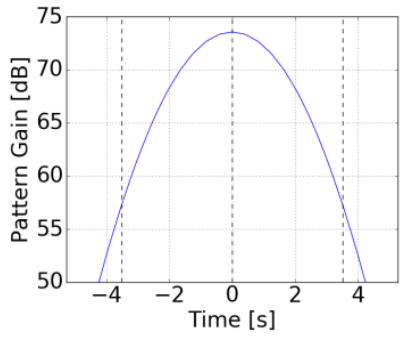

(b)
Fig.8: Example azimuth patterns shown as a function of the azimuth time. The vertical lines highlight the time limits of two PRI cycles. (a) Planar antenna of $5.0 \mathrm{~m}$ length with a sinc pattern (A height of $1.3 \mathrm{~m}$ is assumed to compute the maximum gain). (b) Reflector antenna of TABLE I, azimuth cut at the center of the swath.

The pattern in Fig.8 (a) is a sinc pattern from a directly radiating array with a length of $5 \mathrm{~m}$. Typically, for a SAR system, such an aperture would be enough to achieve a $2.5 \mathrm{~m}$ resolution, in the absence of Doppler spectral gaps. Recalling the worst-case in Fig.7 (a), it is clear that, in the slow PRI variation mode, two looks of $5 \mathrm{~m}$ could be achieved instead with the same antenna. The pattern in Fig.8 (b) is that of the reflector design of TABLE I, taken at the center of the swath.

The results of the analysis of the ENL for the sinc pattern (Fig.8 (a)) are shown in Fig.9. Two quantities are shown, first the Effective Number of Looks (ENL), defined with respect to a look with a flat pattern showing the maximum gain and the appropriate bandwidth. Second, the gain in ENL (translating to signal power) from using the whole available Doppler bandwidth (assumed to be constrained to $\left[-P R F_{\text {mean }} / 2, P R F_{\text {mean }} / 2\right]$ ), in comparison to the use of a single burst (cycle of PRIs), which is the minimum to achieve full resolution. Note that this ENL-gain is only possible if the full Doppler bandwidth is broadcast to ground, which has implications for the system's data rate. It should be stressed that a better gain does not imply a better performance, as the gain of the pattern is not considered, only the ratio between the power in the total available bandwidth and that of a single burst. This figure is meant as a reference to show the possible gain in terms of the ENL attainable by broadcasting the whole Doppler support to ground, as opposed to applying some form of on-board filtering.

The quantities are shown first as a function of the range along the swath - parameterized by the Doppler centroid case (worst / best). Note that the best case and the worst case coincide for the ranges in which no gaps are present. Both quantities are also represented as a function of the target position, represented in 
terms of the azimuth shift in time with respect to the worst-case position (gap at zero Doppler). A shift of zero means the worst case, whereas a shift of $\pm T_{\text {cycle }} / 2$ means the best case. The symmetry of the plots reflects the symmetry of the antenna patterns with respect to the azimuth angle axis.

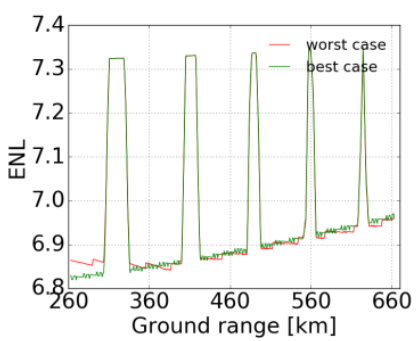

(a)

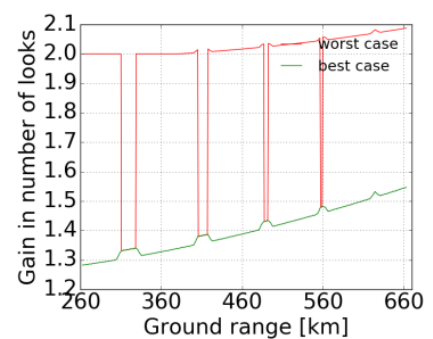

(c)

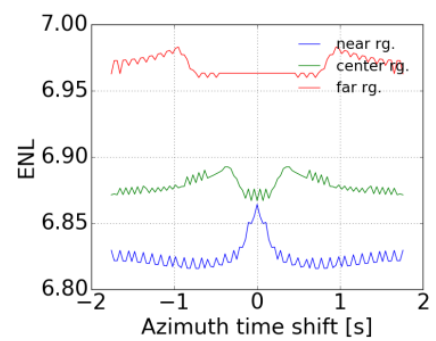

(b)

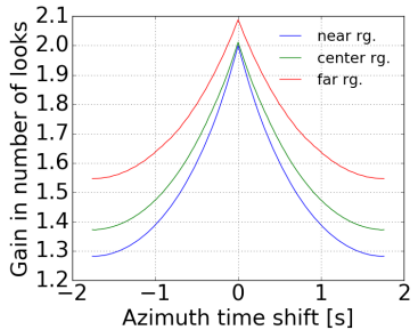

(d)
Fig.9: Analysis of the number of looks achievable for the sinc pattern of Fig.8 (a). (a) Effective number of looks (ENL) shown for the worst (red) and best (green) cases as a function of range. (b) ENL for the near (blue) / center (green) / far (red) ranges of the swath as a function of the target position, represented in terms of the azimuth time shift. A shift of zero corresponds to the worst case whereas a shift of $\pm T_{\text {cycle }} / 2$ (extremes of the shift axis) corresponds to the best case. (c) Gain in the number of looks - ratio of the ENL obtained from a single burst to that using the Doppler bandwidth within $P R F_{\text {mean }}$ - as a function of range, parametrized by the Doppler shift case. (d) Gain in the number of looks as a function of the target's azimuth time shift (along-track position), parametrized by the range position.

The plots indicate that the difference between the best and the worst case is small in terms of the ENL, due to the wide pattern illumination. In Fig.9 (a), the ENL of the worst case (highest Doppler centroid, gap at zero Doppler) is actually slightly better than that of the best case (no Doppler centroid) in very near range. The reason is that the ENL is computed by integrating the pattern power within $\left[-P R F_{\text {mean }} / 2, P R F_{\text {mean }} / 2\right]$, and in this scenario the worst case has a single gap at the center (the gaps of the next cycles fall outside the PRF and do not matter for the computation), whereas the best one has two gaps in regions whose gain is about $-2.5 \mathrm{~dB}$ below the peak. The ENL is better at far range (assuming gaps) because the gap duration gets smaller (cf. Fig.4 (b)) and is seen not to be very sensitive to the target positions shift, for a given range (cf. Fig.9 (b)). As indicated in Fig.9 (c, d), the gain is roughly a factor of two for the worst case, whenever gaps are present (though the performance is better without gaps!), and smaller for the best one, showing moderate sensitivity to range.

A similar analysis was performed for the reflector design in TABLE I, as shown in Fig.10. In this case, some of the effects seen in Fig.9 are also visible. The main differences are due to the fact that the patterns are narrower in azimuth (cf. Fig.8) and that the reflector's patterns are not separable in azimuth and range.
This means that the shape of the azimuth cuts change, showing a broadening due to the defocusing effect near the swath edges. This explains why the number of looks is reduced again in far range, and the expected improvement is not seen.

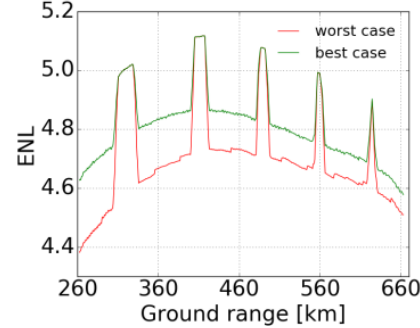

(a)

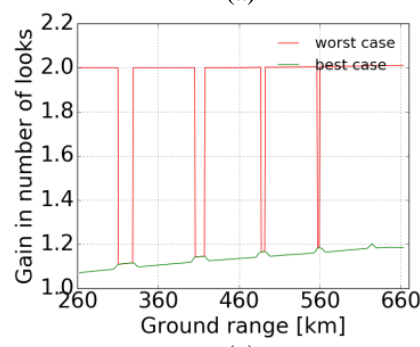

(c)

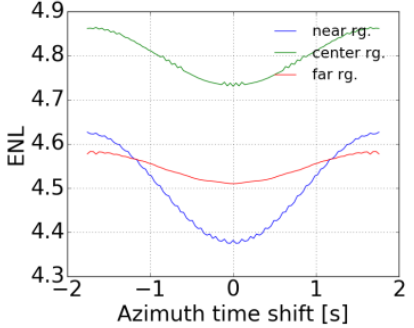

(b)

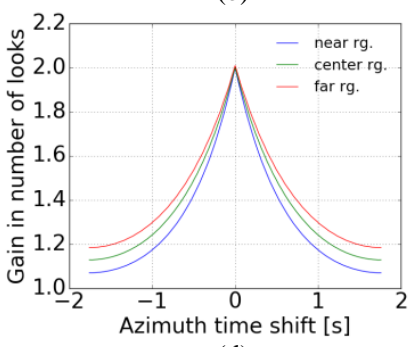

(d)
Fig.10: Analysis of the number of looks achievable for the reflector pattern of TABLE I (Fig.8 (b) applies for the center range of the swath). (a) ENL as a function of range, parametrized by the Doppler shift case. (b) ENL as a function of the target position / shift, parametrized by the range position. (c) Gain in the number of looks as a function of range, parametrized by the Doppler shift case. (d) Gain in the number of looks as a function of the target's azimuth time shift (along-track position), parametrized by the range position.

\section{MODE VARIATIONS AND OTHER MisSION ASPECTS}

Having established the mode's main timing aspects, this section considers add-ons and variations of the mode assuming the availability of multiple azimuth channels. Motivated by the signal's Doppler spectral properties, an azimuth antenna pattern design and azimuth DBF concept are proposed in Section III.A. Section III.B discusses the possibility of steering the beam in azimuth during the acquisition and the implications of this strategy for the SAR performance.

Next, other aspects of the mode's performance are considered. Section III.C briefly addresses the properties of the range ambiguous signals.

\section{A. Azimuth Antenna Patterns and Multichannel Beamforming Concept}

As may be inferred from the analysis of the spectra, the maximum Doppler centroid (proportional to $T_{g a p}$ and thus to $\Delta_{\text {cycle }}$, according to (16)) and the azimuth antenna pattern play an important role for the system's performance. In order to achieve the intended resolution and adequate signal-to-noise ratio (SNR) for all positions over azimuth, the pattern should be made broad enough so that the spectrum has sufficient gain in the (high-squint) worst case. Thus, as is also the case in ScanSAR, the design of the antenna's beam over azimuth must match constraints imposed by the timing. The requirement of broad patterns in azimuth leads to short apertures with lower gain, which can be compensated by the 
use of reflector architectures and suitable azimuth beamforming. A suitable DBF alternative is described in the following.

The rationale for the current DBF concept is that - since the total PRI variation $\triangle P R I$ is moderate and a very long sequence of PRIs is used $\left(N_{P R I} \gg 1\right)$ - the PRIs can be assumed to be nearly constant over a short time window. For instance, for the parameters of TABLE II, the PRI step between adjacent pulses is in the order of $0.7 \mathrm{~ns}$. This means the azimuth sampling is locally regular. This greatly facilitates the use of frequency-domain techniques for digital beamforming, provided that the system possesses multiple digitized azimuth channels (each of them assumed an available data stream). For instance, a short-time FFT over azimuth with a small number of pulses (e.g. 8 or 16) could be used to efficiently implement the subdivision of the Doppler spectrum into an equivalent number of sub-bands, as illustrated in Fig.11.

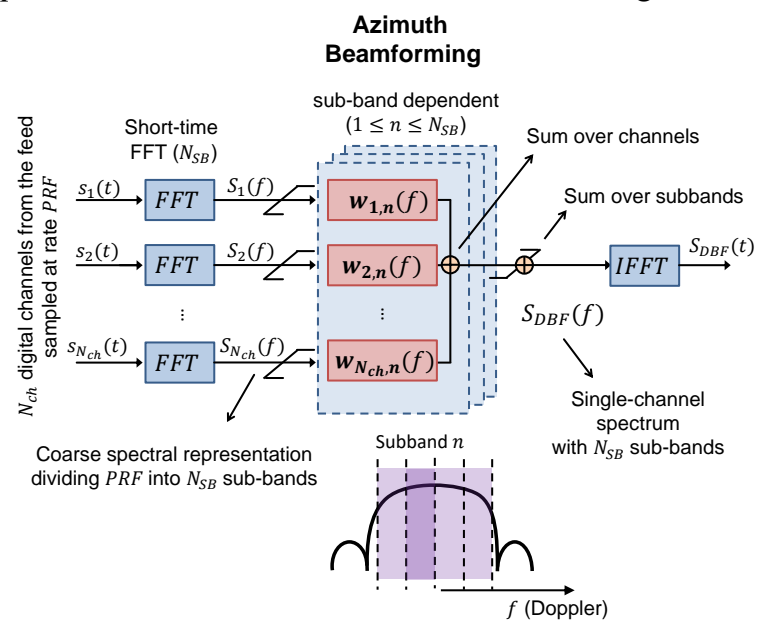

Fig.11: Block diagram for azimuth Doppler sub-band dependent DBF. The bands are separated using a short-time FFT and each sub-band undergoes DBF independently. Each $s_{k}(f), k \leq N_{c h}$, is used $N_{S B}$ times with different $w_{k, n}(f)$.

An alternative scheme is a bank of band-pass finite impulse response (FIR) filters, as illustrated in Fig.12. The relative complexity and attractiveness of each architecture depends on the concrete implementation strategy. However, one interesting aspect of the FIR architecture is its applicability to other modes, enabling the system to operate in multiple imaging modes with just a change in parameters, as will be justified next.

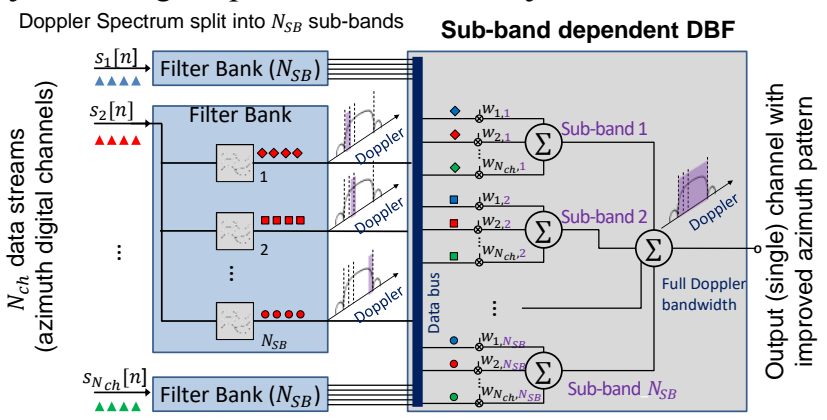

Fig.12: Alternative block diagram for azimuth Doppler sub-band dependent DBF. The bands are separated using a bank of $N_{S B}$ band-pass filters, the same for every channel in azimuth (whose samples are represented in different colors). Each subband (the output of a particular filter, whose samples are represented by different shapes) undergoes the DBF independently. The final summation restores the full Doppler bandwidth.
Let the input signal of the $i^{\text {th }}$ channel be denoted $s_{i}[n]$, where $n$ denotes the sample index. Further, assume that the same set of FIR filters are used for all channels, with coefficients $h_{m, k}$ for the $m^{\text {th }}$ sub-band ( $k$ denotes the tap index). Then, the output of the $m^{\text {th }}$ filter, having the $i^{\text {th }}$ channel data as input is

$$
y_{m, i}[n]=\sum_{k=1}^{N_{\text {taps }}} s_{i}[n-k] \cdot h_{m, k},
$$

as indicated in the expanded block diagram of Fig.13.
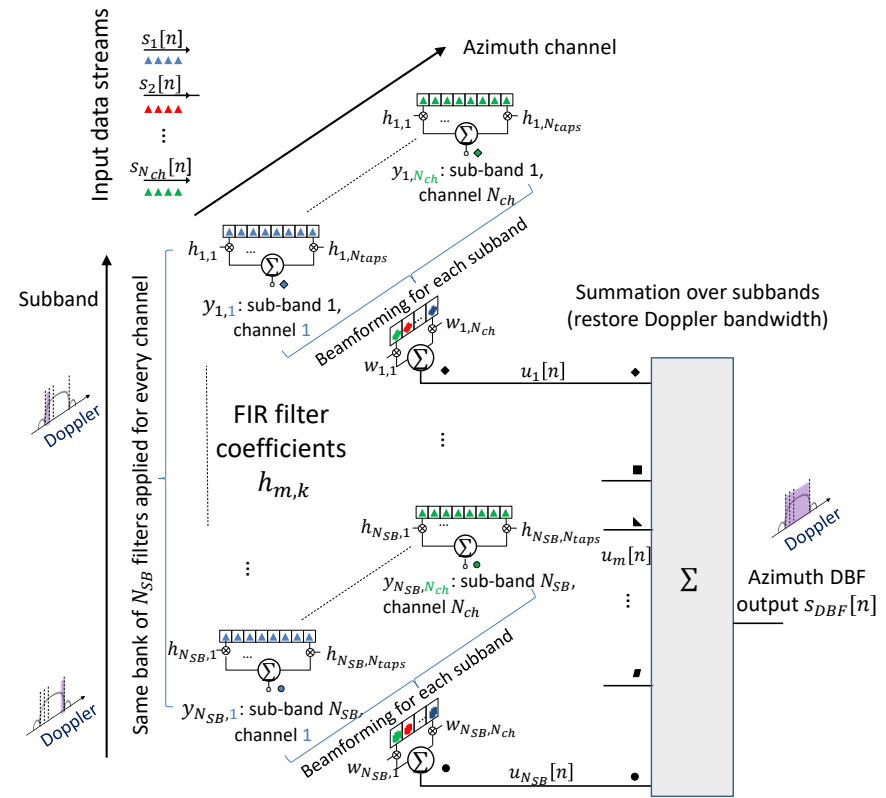

Fig.13: Expansion of the FIR architecture's block diagram. Input samples are processed over the same bank of $N_{S B}$ filters with $N_{\text {taps }}$ for each channel, and then combined over the channels with specific weights for each sub-band. The final summation restores the full Doppler bandwidth support. The input samples are indicated by triangles and the output of a given sub-band by different shapes. Each azimuth channel is represented in a specific color, and their final combination in black.

In a second step, the signals are combined with beamforming weights $w_{m, i}$, with $m$ denoting the sub-band and $i$ the channel, to yield $N_{S B}$ signals

$$
u_{m}[n]=\sum_{i=1}^{N_{c h}} y_{m, i}[n] \cdot w_{m, i}
$$

Finally, the sub-bands are combined to recover the full Doppler support, yielding

$$
\begin{aligned}
s_{D B F}[n] & =\sum_{m=1}^{N_{S B}} \sum_{i=1}^{N_{c h}} y_{m, i}[n] \cdot w_{m, i} \\
s_{D B F}[n] & (20) \sum_{m=1}^{N_{S B}} \sum_{i=1}^{N_{c h}} \sum_{k=1}^{N_{\text {taps }}} s_{i}[n-k] \cdot h_{m, k} \cdot w_{m, i} \\
= & \sum_{i=1}^{N_{c h}} \sum_{k=1}^{N_{\text {taps }}} s_{i}[n-k] \cdot\left(\sum_{m=1}^{N_{S B}} h_{m, k} \cdot w_{m, i}\right) \\
s_{D B F}[n]= & \sum_{\text {ch }}^{N_{\text {taps }}} \sum_{i=1} s_{k=1}[n-k] \cdot w_{D B F}[i, k] .
\end{aligned}
$$


The factorization of the coefficients in (22) shows that it is not necessary to actually implement $N_{c h}$ banks of $N_{S B}$ filters. Due to the assumption that the filters are the same for each sub-band, only $N_{c h}$ filters, each with $N_{\text {taps }}$ complex coefficients $w_{D B F}[i, k]$ are needed. Note that this is also what is necessary to implement the azimuth beamforming for a multichannel staggered SAR system [17], or a single channel staggered SAR. That is to say, provided that the filter maximum length is large enough, an update of the weight look-up table allows the same hardware architecture to operate in any of these modes. Moreover, the low order interpolation (for the simulation examples two-point linear interpolation is used) required to resample the data acquired with the slow PRI variation to a regular azimuth grid can be incorporated into the weights $w_{D B F}$ to yield the resampled data.

In either architecture, each of the sub-bands can receive different beamforming weights over azimuth. After combination over the azimuth channels and either inverse short-FFT over the azimuth samples (Fig.11) or summation (Fig.12), a Doppler-dependent weighting is achieved. This bears the potential of improving system performance, at the cost of increased complexity due to the multichannel architecture. The Minimum Variance Distortionless Response (MVDR) beamformer (cf. [24], [25]), akin to a matchedfilter, using the average of the patterns over the sub-bands as reference is an interesting approach to maximize the gain ${ }^{5}$. Should oversampling (in terms of the PRF of a single channel) allow it, bands outside the required bandwidth may also be suppressed.

The patterns obtained in near range by applying this approach with $N_{S B}=16$ for the system of TABLE I are shown in Fig.14, where it is compared to the Linearly Constrained Minimum Variance (LCMV) used in the single-channel case of Section IV.A to control the beamwidth. Aliasing is taken into account, and the Doppler regions spaced by integer multiples of $P R F$ receive the same weights. The improvement in the patterns is clear, both in terms of the increase in gain $(0.5$ to $5 \mathrm{~dB})$ and sidelobe suppression. The latter is a consequence of the weight mismatch for the aliased bands, which produces a convenient "anti-aliasing" effect on the Doppler spectrum.

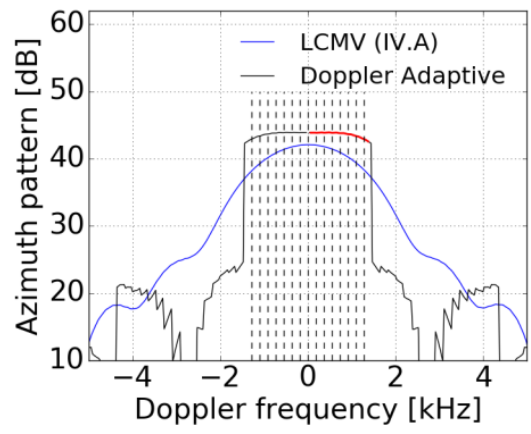

Fig.14: Azimuth patterns comparing the LCMV beamformer and the proposed Doppler-adaptive beamforming in near range, for the system of TABLE I, assuming a mean PRF of $2917.5 \mathrm{~Hz}$. The portion of the spectrum processed for the worst case is highlighted in red (positive Doppler).

\footnotetext{
${ }^{5}$ Note that in this case no sampling rate expansion or reconstruction is performed on the multichannel data, but rather the multiple channels are employed to improve the azimuth pattern gain on a sub-band basis. This is, more sophisticated that e.g. the in-cycle steering of a single-channel TOPS, which is why the best-case performance is not degraded.
}

\section{B. Azimuth Steering in Rx: TOPS (Aft to Fore Steering)}

The proposed Doppler adaptive beamforming concept exploits the slow PRI variation to apply frequency domain beamforming techniques which improve the pattern characteristics. The result is a pattern which is still broad, but with better gain and sidelobe characteristics. As discussed in Section II.B, the broad patterns in azimuth show considerable potential in terms of multilooking, but the achieved illumination is inefficient, if a single fullresolution look is used.

A different illumination strategy would be to relinquish the excess bandwidth (and thus the possibility of multilooking) by narrowing the illumination in azimuth. A time-varying steering of the azimuth pattern within the PRI cycle is one possibility to do so, as schematically illustrated in Fig.15. It should be noted that the gap position varies with range, and therefore the concept relies on digital beamforming on receive to achieve a rangeadaptive steering. The time referential in Fig.15 thus applies to a generic range $R_{0}$, and the steering is assumed range-adaptive.

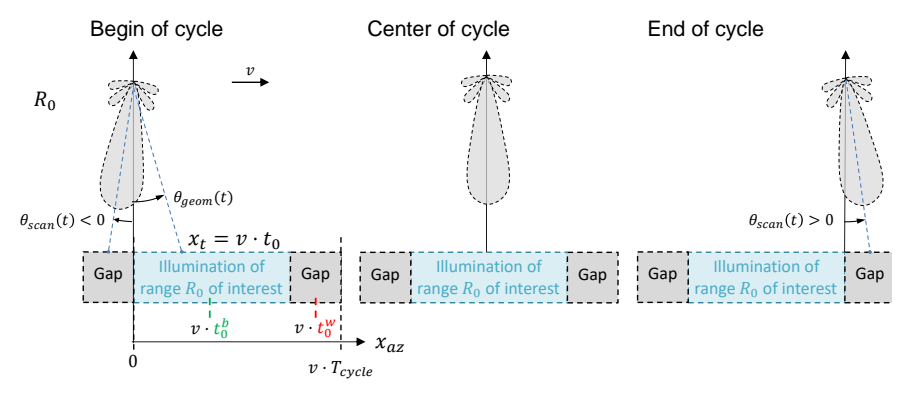

Fig.15: Azimuth scan of patterns during cycle, to improve the illumination efficiency. The antenna main beam in azimuth is steered to the position of the worst-case target (center of gap) in the beginning of the cycle and $\theta_{\text {scan }}(t)$ increases linearly during it, so that it is steered to the best-case target in the center of the cycle. Consequently, the next gap is illuminated at the end of it.

For a generic target position $x_{t}=v \cdot t_{0}$, the geometric azimuth angle between target and platform (which defines the Doppler frequency) is (assuming a simplified flat-Earth model) $\theta_{\text {geom }}\left(t, t_{0}\right) \cong-v / R_{0} \cdot\left(t-t_{0}\right)$.

The steering is described by a linearly varying scan angle

$$
\theta_{\text {scan }}(t)=k_{\text {scan }} \cdot\left(t-T_{\text {cycle }} / 2\right)
$$

and the azimuth angle with which a target is seen by the antenna (which defines the Doppler spectral weighting) is then

$$
\begin{aligned}
& \theta_{a z}\left(t, t_{0}\right)=\theta_{\text {geom }}\left(t, t_{0}\right)-\theta_{\text {scan }}(t) \\
& \theta_{a z}\left(t, t_{0}\right) \cong-\frac{v}{R_{0}} \cdot\left(t-t_{0}\right)-k_{\text {scan }} \cdot\left(t-\frac{T_{\text {cycle }}}{2}\right) .
\end{aligned}
$$

The angular span (variation of $\theta_{a z}\left(t, t_{0}\right)$ ) over the cycle is then

$$
\Delta \theta_{a z} \cong \frac{v}{R_{0}} \cdot T_{i l l} \cdot\left(1+\frac{R_{0}}{v} \cdot k_{\text {scan }}\right)=\frac{v}{R_{0}} \cdot T_{i l l} \cdot \beta_{\text {scan }}
$$


where $\beta_{\text {scan }}$ is the factor by which the "geometrical" angular span (the variation due to $\theta_{\text {geom }}\left(t, t_{0}\right)$ ) is modified. $\beta_{\text {scan }}$ may lead either to a compression or expansion of the azimuth patterns, depending of the sign and magnitude of $k_{\text {scan }}$.

i) For $k_{\text {scan }}>0$ (which means $\theta_{\text {scan }}(t)<0$ in the first half of the cycle, cf. (33)), the antenna starts looking backwards and ends the cycle looking forward, which corresponds to the TOPS mode [26] (aft to fore steering, as in Fig.15). In this case, $\beta_{\text {scan }}>1$ and the patterns are compressed, in the sense that a larger angular span is covered in the same time.

ii) For $-v / R_{0}<k_{\text {scan }}<0,0<\beta_{\text {scan }}<1$ and one has a sliding spotlight [22] with pattern expansion. The limit case of $\beta_{\text {scan }}=0$ corresponds to the starring spotlight, and a single view angle for a particular target.

iii) For $-2 \cdot v / R_{0}<k_{\text {scan }}<-v / R_{0}$, one has the expansion factor $-1<\beta_{\text {scan }}<0$, meaning the patterns are "inverted" and still expanded.

iv) The case $k_{\text {scan }}<-2 \cdot v / R_{0}$ is in turn an inverse-TOPS [27] (fore to aft steering), with $\beta_{\text {scan }}<-1$. In this case, the patterns are "inverted" and again compressed, in comparison to the non-steering antenna.

In accordance with the case described in Fig.15, forcing the steering to point to the previous worst-case target at the cycle begin, i.e., $\theta_{a z}\left(0,-T_{\text {gap }} / 2\right)=0$, leads to

$$
k_{\text {scan }}=\frac{v}{R_{0}} \cdot\left(\frac{T_{\text {gap }}}{T_{\text {cycle }}}\right)
$$

and thus

$$
\beta_{\text {scan }}=1+\frac{T_{\text {gap }}}{T_{\text {cycle }}}
$$

confirming that the proposed scanning constitutes a TOPS mode, with azimuth patterns which are compressed with respect to the non-scanning case, and the SAR performance variation between the worst and the best case is expected to be reduced. The Dopplertime plane for this scanning is illustrated in Fig. 16.

It can be seen from the plot that every target is at the main beam of the azimuth antenna pattern at a given point in time. This constitutes the main advantage of this strategy. The compression of the patterns by a factor $\beta_{\text {scan }}>1$ means, however, that the azimuth resolution may be worsened, as the effective beamwidth is reduced.

An inverse-TOPS mode, with properties which are similar to conventional TOPS, is equally possible by inverting the scan and setting $\theta_{a z}\left(0, T_{i l l}+T_{\text {gap }} / 2\right)=0$, but the conventional TOPS timeline is preferred due to the reduced magnitude of $k_{\text {scan }}$.

\section{A Note on Range Ambiguities}

Operation of a system with PRI variation may considerably impact range ambiguities [28], especially in the case of a fast variation. Assuming distributed targets, the SAR signal from two non-overlapping resolution cells is expected to be uncorrelated.

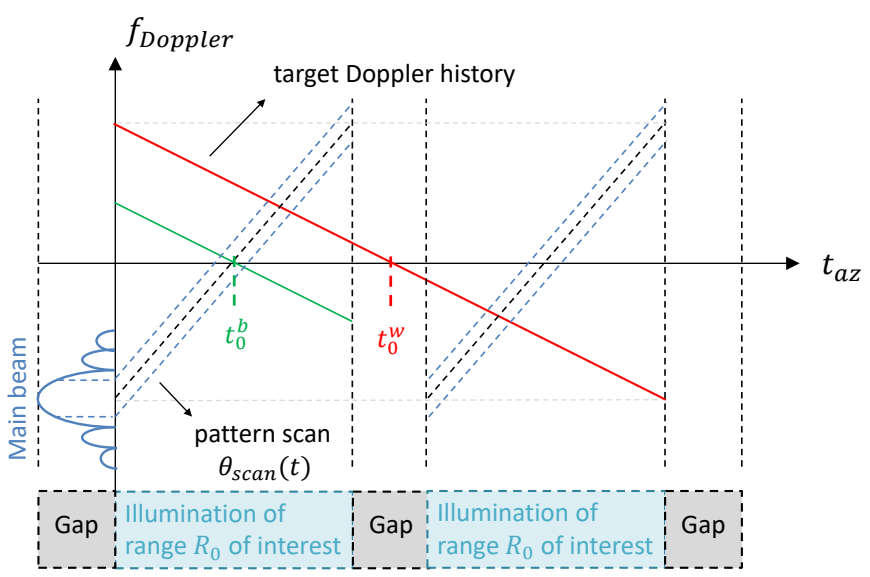

Fig.16: Doppler-time plane for azimuth scan of patterns during cycle. The Doppler history of targets is assumed to be linear, with the zero-Doppler time depending on the target position: $t_{0}^{b}$ and $t_{0}^{w}$ refer to the minimum (best-case) and maximum (worstcase) squint scenarios. The steering modifies the spectral weighting due to the azimuth antenna pattern. Note that the worst-case target is within the main beam in the end of the cycle, but also at the beginning of the next cycle, meaning two looks are still available for this particular case. The best-case target is seen in the main beam in the center of the steering.

This effect manifests in two ways which are relevant for this analysis:

A) In azimuth: as expected, the SAR signal will decorrelate over the flight direction, at a rate depending on the antenna length. This affects the main signal and the range ambiguities likewise.

B) In range: the same is true if the resolution cell migrates over range. This does not affect the main signal, but the changes in the PRI with time impose corresponding changes in the position of the ambiguities from pulse to pulse.

The point is then to analyze whether effect B) is fast enough to overshadow A) or not. In order to quantify this phenomenon, take the transmitted SAR signal to be:

$s_{T x}(t)=s_{\text {chirp }}(t) \cdot \exp \left(j \cdot 2 \cdot \pi \cdot f_{0} \cdot t\right)$,

where $f_{0}$ is the carrier frequency and $s_{\text {chirp }}(t)$ is the chirp waveform. The received signal, accounting for the two-way travel time delay and after demodulation to baseband, can be expressed as

$$
\begin{aligned}
s_{b b}(t, r)=k_{a t t}(r) \cdot a(\theta(r)) \cdot \sigma(r) & \cdot s_{\text {chirp }}\left(t-\frac{2 \cdot r}{c}\right) \\
\cdot & \exp \left(-j \cdot \frac{4 \cdot \pi}{\lambda} \cdot r\right),
\end{aligned}
$$

consisting of $s_{T x}(t)$ delayed by $2 \cdot r / c$; modified by rangedependent factor and demodulated with the conjugate of the carrier. The range dependent factors include a free-space attenuation $k_{a t t}(r)$, a modulation by the antenna pattern $a(\theta(r))$ and a complex contribution from the target at $r$, expressed by $\sigma(r)$.

The position of the range ambiguities with respect to the signal is closely to the definition of the delays $d_{i, k}$ in (9). In fact, it can be shown that the signal from the $n^{t h}$ order range ambiguity related to the $i^{\text {th }}$ transmit pulse is given by 


$$
\begin{aligned}
& s_{r g-a m b}(t, r ; n, i)=s_{b b}\left(t, r-\frac{c}{2} \cdot d_{i, n}\right) \\
& s_{r g-a m b}(t, r ; n, i)=k_{a t t}\left(r-\frac{c}{2} \cdot d_{i, n}\right) \cdot a\left(r-\frac{c}{2} \cdot d_{i, n}\right) \\
& \cdot \sigma\left(r-\frac{c}{2} \cdot d_{i, n}\right) \cdot s_{c h i r p}\left(t-\frac{2 \cdot r}{c}+d_{i, n}\right) \\
& \cdot \exp \left(-j \cdot \frac{4 \cdot \pi}{\lambda} \cdot r\right) \cdot \exp \left(j \cdot 2 \cdot \pi \cdot f_{0} \cdot d_{i, n}\right) .
\end{aligned}
$$

The terms of (30) indicate that the attenuation $k_{a t t}(r)$ and the antenna pattern $a(r)$ are changed by the delay, as expected. Furthermore, the chirp waveform is delayed and the exponential of $-j \cdot 4 \cdot \pi \cdot r / \lambda$, related to the main signal's position in (29) is still present. The other two terms are discussed next.

The term $\sigma\left(r-c / 2 \cdot d_{i, n}\right)$ describes the contribution of the area on ground to the ambiguous energy. In order to analyze whether the range ambiguities arise from a different resolution cell or not, define the step between two adjacent PRIs as

$$
P R I_{\text {step }}=\frac{\Delta P R I}{N_{P R I}-1} .
$$

Further, note that (30) implies that the migration of the range ambiguities of order $k$ (a function of $i$ and $k$ ) between adjacent pulses is given by $c / 2 \cdot\left(d_{i+1, k}-d_{i, k}\right)$. It follows directly from (10) that

$$
d_{i+1, k}-d_{i, k}=\left\{\begin{array}{cc}
k \cdot P R I_{\text {step }} & (\text { region } I) ; \\
-P R I_{\text {step }} \cdot\left(N_{P R I}-k\right) & (\text { region } I I) .
\end{array}\right.
$$

From which the migration rate of the ambiguities is a function of the ambiguity order and the step between subsequent PRIs. Recall that for the slow PRI variation $N_{P R I} \gg k_{\max }$ and thus region $I$ is dominant, whereas region $I I$ is a short transient. In this region, the number of pulses required for the migration of the first-order range ambiguities $(k=1)$ - which typically contribute the most to the ambiguous energy - to leave the resolution cell and thus cause the ambiguities to decorrelate is given by

$$
N_{\text {decorr }}=\frac{\delta_{r g} \cdot \frac{2}{c}}{P R I_{\text {step }}}
$$

where $\delta_{r g}$ is the range resolution. The corresponding azimuth displacement can be estimated as

$$
\Delta x_{\text {decorr }}=N_{\text {decorr }} \cdot \frac{v}{\overline{P R F}},
$$

where $v$ is the platform velocity. From [29], [30], the SAR signal in azimuth has a correlation length which is roughly given by the antenna (or aperture) along-track dimension $L_{a z}$. As long as $\Delta x_{\text {decorr }} \gg L_{a z}-$ which tends to be the case for a slow PRI variation - the decorrelation effect of the range ambiguities may be disregarded (Effect A) dominates over B)). For instance, with the parameters of TABLE II, $\triangle P R I=-11.18 \mu \mathrm{s}$, and thus $P R I_{\text {step }}=-0.92 \mathrm{~ns}$. Assuming a $5 \mathrm{~m}$ range resolution, $N_{\text {decorr }} \cong 36$ pulses and $\Delta x_{\text {decorr }} \cong 78.5 \mathrm{~m}$. The range ambiguities can therefore be estimated for an equivalent constantPRF SAR operated at e.g. $\overline{P R F}$. Note that this is a conservative assumption, as the decorrelation tends to improve RASR levels, i.e. suppress the ambiguities. This is true as uncorrelated ambiguities have a flat Doppler spectrum, whereas the spectrum of correlated ambiguities follows the shape of the antenna pattern. This is relevant for RASR levels due to the filter's low-pass characteristic. In the case of a flat spectrum, typically more energy is rejected by the processed bandwidth filter than in the case of correlated ambiguities, whose spectrum has the bulk of their energy concentrated in the main beam. The signal's spectrum is nonetheless the same in both cases.

The final term in (30) is the complex exponential $\exp \left(j \cdot 2 \cdot \pi \cdot f_{0} \cdot d_{i, n}\right)$, which reveals an interesting effect, similar to the known Azimuth Phase Coding (APC) [31], [32]. From (10), (32), an order-dependent phase modulation in azimuth of the ambiguities is caused by the PRI variation. For a fixed $k$, phase ramp varying linearly with $i$ will be formed, which is formally similar to APC. Unlike in APC, however, this does not correspond to a linear phase ramp with respect to azimuth time (a quadratic function of $i$ in this case due to the PRI variation). Therefore, it does not correspond exactly to a constant shift of the Doppler spectrum.

In conclusion, it is important to recognize that the azimuth spectral behavior of the range ambiguities is different in this case than in staggered SAR, i.e. fast PRI variation. For the same PRF, the latter would be less affected by the ambiguities, even though in practice the slow PRI variation is typically more likely to be applied at a coarser azimuth resolution and require less oversampling, and the resulting lower PRF may overshadow this aspect.

\section{Implications for Interferometry}

In this sub-section, a number of possible difficulties which may arise in the interferometric operation of the slow PRI variation mode are considered. The goal is to provide a first-order assessment, in order to quantify potential issues.

The first two aspects addressed in this section are related to two sources of spectral decorrelation. It is a well-known SAR signal property that independent looks are uncorrelated [1] and thus interferometry requires a common Doppler spectrum, with decorrelation ensuing from spectral shifts [33], [34]. In modes with temporal spectral variation (which includes ScanSAR and TOPS), timing mismatches between the master and slave acquisition thus translate into decorrelation. Two sources are illustrated in Fig.17 and considered in the following.

\section{1) Spectral Decorrelation due to Burst (Cycle) Synchronization}

As illustrated in Fig.17 (a), a synchronization error $\Delta t_{\text {syn }}$ between the PRI cycles of master and slave would lead to a cyclical shift of the gap positions. For a fixed range, this changes the position of the gap by a corresponding amount (cf. Fig. 17 (c)). In this case, the amount of spectral shift relates directly to the Doppler rate, that is

$$
\Delta f_{\text {max }}=\max \left(\Delta t_{\text {syn }}\right) \cdot \xi_{\text {Dop }}
$$

where $\xi_{D o p}=-2 \cdot v^{2} /\left(\lambda \cdot R_{0}\right) \cong-570 \mathrm{~Hz} / \mathrm{s}$ for the parameters of TABLE I (at far range). This implies the same synchronization requirement of a ScanSAR acquisition, which is a bit more stringent than in TOPS [35], but which in any case does not pose a challenge. Current spaceborne missions like TerraSAR-X and Sentinel-1 achieve accuracies in the order of $5 \mathrm{~ms}$ or better in the 
burst synchronization, which would lead to a spectral decorrelation of $0.4 \mathrm{~Hz}$ for the suggested L-band system, hence having a negligible impact. It should be pointed out that, as discussed in [35], $\Delta t_{\text {syn }}(t)$ actually varies across the acquisition, according to the properties of the orbital tube. Though this effect is not relevant for a Sentinel-1-like configuration it should be kept in mind, especially for larger orbital tubes and higher frequencies.

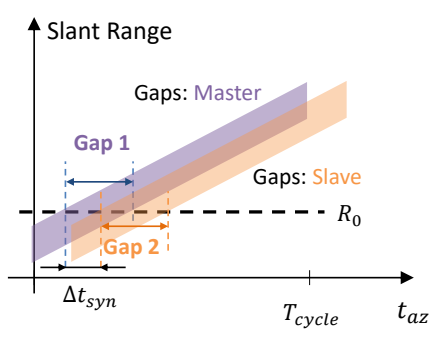

(a)

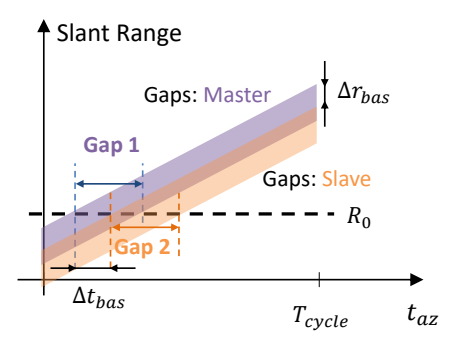

(b)

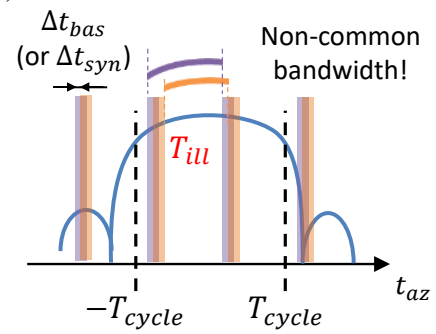

(c)

Fig.17: Interferometric spectral decorrelation due to timing mismatches between master and slave acquisition. (a) PRI cycle synchronization error $\Delta t_{\text {syn }}$ (exaggerated), shifting the position of a given gap. (b) Effect of baseline $\Delta r_{\text {bas }}$, inducing a similar effect. (c) Illustration of Doppler bandwidth mismatch due to a cyclic shift in the gap position, which applies to the two latter cases.

\section{2) Spectral Decorrelation due to Baseline}

As illustrated in Fig.17 (b), the baseline, and in particular the parallel baseline $\Delta r_{b a s}$, between master and slave acquisitions operated with exactly the same PRI sequence induces a slant range shift of the gap pattern. This in turn shifts the position of the gaps by an amount $\Delta t_{\text {bas }}$, which again introduces an azimuth spectral mismatch (cf. Fig.17 (c)). From (5), the inclination of the gaps is a function of the order $k$ (related to the number of pulses transmitted during the two-way travelling time, in the interval $\left[k_{\min }, k_{\max }\right]$ determined by the swath limits):

$$
\frac{d R}{d t}(k)=\frac{c}{2} \cdot k \cdot \frac{\Delta P R I}{T_{\text {cycle }}},
$$

and thus one obtains, for the least steep case $k=k_{\text {min }}$,

$$
\Delta t_{\text {bas }}=\Delta r_{\text {bas }} \cdot \frac{2 \cdot T_{\text {cycle }}}{c \cdot k_{\text {min }} \cdot \Delta P R I} .
$$

From (6), (16), and substituting into (37)

$$
\begin{aligned}
& \Delta P R I=\frac{\tau_{P}}{k_{\max }} \cdot \frac{1-d c_{\text {min }}}{d c_{\text {min }}} \Rightarrow \\
& \Delta t_{\text {bas }}=\Delta r_{\text {bas }} \cdot \frac{2}{c} \cdot \frac{k_{\text {max }}}{k_{\text {min }}} \cdot \frac{T_{c y c l e}}{1-d c_{\text {min }}} \cdot \overline{P R F},
\end{aligned}
$$

where $d c_{\text {min }}$ is the minimum pulse duty cycle (cf. (16) and footnote 2).
According to the discussion in Section II.B (cf. Fig.6), the gaps translate into Doppler centroids following the opposite inclination as the Doppler rate $\xi_{\text {Dop }}$. The final Doppler shift as a function of the parallel baseline $\Delta r_{\text {bas }}$ (line-of-sight projection) is

$$
\Delta f_{\text {max }}=\Delta r_{\text {bas }} \cdot \frac{2}{c} \cdot \frac{k_{\text {max }}}{k_{\min }} \cdot \frac{T_{\text {cycle }}}{1-d c_{\min }} \cdot \overline{P R F} \cdot \xi_{\text {Dop }}
$$

Using the parameters from TABLE I and TABLE II, this amounts to a worst-case shift of about $0.068 \mathrm{~Hz} / \mathrm{m}$. Or in other words, the shift would exceed $5 \%$ of the processed bandwidth $(75 \mathrm{~Hz}$ ) for a baseline larger than $1100 \mathrm{~m}$. Moreover, the spectral shift varies within the swath, as the Doppler rate $\xi_{\text {Dop }}$, the gap order $k$ and the parallel baseline $\Delta r_{b a s}$ are functions of range. The above equations can be written in terms of the horizontal baseline $\Delta r_{\text {hor }}$ (fixed value, no line-of-sight projection) to highlight this:

$$
\Delta f\left(R_{0}\right)=\Delta r_{\text {hor }} \cdot \frac{2}{c} \cdot \frac{T_{\text {cycle }}}{\Delta P R I} \cdot\left(\frac{\xi_{\text {dop }} \cdot \sin (\phi)}{k}\right)\left(R_{0}\right),
$$

where the terms between brackets are implicit functions of range and $\phi$ is the incidence angle. In this case, $\xi_{d o p} / k$ decreases with range, whereas $\sin (\phi)$ increases, an effect which introduces a compensation. Using the same parameters as above, the shift ratio varies between [-0.024, -0.027$] \mathrm{Hz} / \mathrm{m}$ when moving from near to far range, even though $\sin (\phi)$ nearly doubles.

A possibility to compensate this effect could be to include a delay in the PRI cycle in order to align the gaps for a reference range. The alignment could be applied for each acquisition by considering the satellite position with respect to the reference orbit, similar as done with the burst synchronization in the previous section.

A secondary effect is that range bins directly before and after the gap will have gaps only for one of the acquisitions. This is however not critical since proper filtering can be applied to the gapless cases to extract the Doppler bandwidth of interest.

\section{3) Azimuth Co-registration of Interferometric Pairs}

As discussed in [34], [36], the combination of a Doppler centroid and an azimuth co-registration error between image pairs can lead to interferometric phase biases. Whereas a squinted Stripmap acquisition shows an unimportant constant phase offset in the presence of misregistration, in modes involving a time-dependent Doppler centroid variation as ScanSAR and TOPS, a time-dependent phase modulation (usually a ramp for a linear Doppler centroid variation) will occur. Assuming a residual misregistration error $\Delta t$ (due to the orbit and other effects as e.g. motion in the scene or different ionospheric delays) between master and slave, the ScanSAR interferometric bias [36], [37] is given by

$\phi_{a z-\operatorname{error}}(t)=2 \cdot \pi \cdot f_{d c}(t) \cdot \Delta t$.

Taking Fig.4 (c) as a reference, $\left|f_{d c}(t)\right|<900 \mathrm{~Hz}$. Assuming a peak-to-peak variation smaller than $2000 \mathrm{~Hz}$, an error smaller than $3^{\circ}$ would require $\Delta t_{\text {orbit }}<4.2 \mu \mathrm{s}$. For an orbit velocity of 7466 $\mathrm{m} / \mathrm{s}$ (matching the orbit height in TABLE I) and assuming a $5 \mathrm{~m}$ 
sampling of the image in azimuth, this represents an orbital accuracy in the order of $3 \mathrm{~cm}$ or 0.006 pixels. According to [36], this is in the order of the best-case 3D $1 \sigma$ accuracy achieved by TerraSAR-X and approximately a factor of 4 looser than the requirements of the TOPS mode described therein. [34] also reports an accuracy requirement of $1 \mathrm{~cm}$ for Sentinel-1 TOPS interferometry, achieved through the procedure described in [36]. Though challenging, this accuracy is deemed achievable with stateof-the-art compensation methods as described in [36], [34], which could be adapted to the slow PRI variation mode by, e.g., exploiting the different looks (see Section II.B.3). Furthermore, the ranges in which no blockage occurs (cf. e.g. Fig.4 (a)), though limited, could provide additional information for the corregistration. Note that, as discussed in [33], the range co-registration is not a concern even for a more demanding TOPS mode with an overall Doppler centroid variation in the order of $10 \mathrm{kHz}$.

\section{L-BAND DESIGN EXAMPLES}

This section presents simulation results to assess the SAR performance of a reflector system in L-Band capable of operating in multiple complementary modes. The system described in TABLE I is based on a $12.0 \mathrm{~m}$ diameter reflector with a 2D multichannel feed, illustrated in Fig.18. In elevation, the feed consists of 40 elements (square patch antennas [38]), spaced at $0.6 \lambda$. In azimuth, 6 elements are summed pairwise to yield 3 channels spaced at $1.0 \lambda$.

\section{Multichannel Feed}

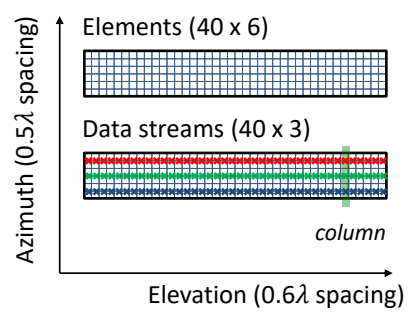

Fig.18: Reflector system's 2D multichannel feed. The channels are assumed to be individually digitized and stored, as required by the multichannel modes, thought the single-channel configurations shown use fixed weighting of the columns and thus could be also implemented with analog weighting in azimuth, reducing the number of analog-to-digital converters by a factor of three.

The reflector system will be applied in different modes, described in the following sub-sections. Sections IV.A and IV.B consider single-polarization acquisitions, whereas Sections IV.C to IV.E address fully polarimetric modes.

Note that the multichannel capability is necessary for the modes in Sections IV.B and IV.E, but the other modes use fixed azimuth beamforming and could be implemented as single-channel systems. A more detailed description of each section is provided in the following.

Section IV.A illustrates the performance of the slow PRI variation mode described in the previous sections, in the case of a $400 \mathrm{~km}$ swath imaged with $5 \mathrm{~m}$ azimuth resolution in single polarization. In this case, the system is operated in a single-channel configuration, applying fixed azimuth weights to the channels.
TABLE I

L-BAND MULTIMODAL REFLECTOR SYSTEM PARAMETERS Platform and swath parameters

\begin{tabular}{|c|c|c|}
\hline Parameter & Symbol & Value \\
\hline Orbit height & $h_{\text {orbit }}$ & $773 \mathrm{~km}$ \\
\hline Orbital velocity & $v_{\text {orbit }}$ & $7466 \mathrm{~m} / \mathrm{s}$ \\
\hline Swath width on ground & $W_{\text {ground }}$ & $400 \mathrm{~km}$ \\
\hline $\begin{array}{l}\text { Swath minimum/maximum } \\
\text { incidence angle }\end{array}$ & $\phi_{\min } / \phi_{\max }$ & $\begin{array}{l}21.0-45.2 \mathrm{deg} \\
\text { (single-pol) } \\
\text { mode-dependent } \\
\text { (quad-pol) }\end{array}$ \\
\hline $\begin{array}{l}\text { Swath minimum/maximum } \\
\text { slant range }\end{array}$ & $R_{\min } / R_{\max }$ & $\begin{array}{l}821.5-1044.1 \mathrm{~km} \\
\text { (single-pol) } \\
\text { mode-dependent } \\
\text { (quad-pol) }\end{array}$ \\
\hline
\end{tabular}

Reflector and feed parameters

\begin{tabular}{lll}
\hline Parameter & Symbol & Value \\
\hline Diameter & $D$ & $12.0 \mathrm{~m}$ \\
Focal length & $F$ & $12.0 \mathrm{~m}$ \\
Feed offset in elevation & $d_{O F F}$ & $8.0 \mathrm{~m}$ \\
Center frequency & $f_{0}$ & $1.2575 \mathrm{GHz}$ \\
Number of channels in & $N_{e l} / N_{a z}$ & $40 / 3$ \\
elevation/azimuth & & \\
Channel spacing in & $d_{e l} / d_{a z}$ & $0.6 \lambda / 1.0 \lambda$ \\
elevation/azimuth & $\theta_{t i l t}$ & $28.7 \mathrm{deg}$ \\
Elevation tilt angle & $L_{\Omega}$ & $2 \mathrm{~dB}$ \\
Feed ohmic losses &
\end{tabular}

Common pulse and $\mathrm{Tx} / \mathrm{Rx}$ hardware parameters

\begin{tabular}{lll}
\hline Parameter & Symbol & Value \\
\hline Noise temperature & $T_{\text {noise }}$ & $650 \mathrm{~K}$ \\
Chirp bandwidth & $B w_{\text {chirp }}$ & $84 \mathrm{MHz}$ \\
& (Except for IV.C:42MHz) \\
\hline \hline
\end{tabular}

The weights are obtained by applying the Linearly Constrained Minimum Variance (LCMV) beamformer [24], [25], [18] to set the required beamwidth. In Section IV.B, the azimuth multichannel concept described in Section III.A is considered, to assess the potential performance gain.

Next, focus is turned to quad-pol operation. In this case, interleaving of $\mathrm{H}$ and $\mathrm{V}$ pulses is required, which lowers the effective PRF of each polarization. As a consequence, achieving acceptably low RASR levels in far range becomes more challenging, since the azimuth performance requirements constrain the minimum feasible PRF. For this reason, the swath is reduced as necessary according to the mode. All swaths start at the same $21^{\circ}$ incidence angle as the single-pol case and are thus a sub-set of the initial swath.

Section IV.C considers a fully polarimetric single-channel slow PRI variation mode. In this case, the design logic of Section II.A is applied with the parameters of the interleaved sequence. A swath of $350 \mathrm{~km}$ is imaged with $10 \mathrm{~m}$ azimuth resolution. The reduction in azimuth resolution allows a narrower azimuth beam to be employed. Thus, a different set of fixed LCMV weights is chosen. In Section IV.D, the same azimuth beamforming is used in a single-channel staggered SAR mode [16] which images a 200 $\mathrm{km}$ swath with $5 \mathrm{~m}$ azimuth resolution (recall that Staggered SAR has no spectral gaps and can thus exploit the full beamwidth), for a comparison with the slowly varying PRI mode, employing the same beam characteristics in azimuth. Section IV.E describes the 
performance of a complementary multichannel staggered SAR mode [17], [18] which could be applied to the same multichannel system of Section IV.B to image the first $200 \mathrm{~km}$ of the swath with $2 \mathrm{~m}$ resolution in quad-pol.

The imaging requirements are azimuth ambiguity-to-signal ratio (AASR) and range ambiguity-to-signal ratio (RASR) better than $-25 \mathrm{~dB}$ for single-pol and $-24 \mathrm{~dB}$ for quad-pol operation. The combination of AASR and RASR should lead to an ambiguity-to-signal ratio (ASR) lower than $-24 \mathrm{~dB}$. The noise equivalent sigma zero (NESZ) levels are required to be better than $-25 \mathrm{~dB}$, regardless of polarization.

\section{A. Single-Pol Slowly Varying PRI Mode: Single-channel in Azimuth}

In a first example, the $12 \mathrm{~m}$ reflector with the multichannel feed of TABLE I is used in a single-channel configuration. The mode's parameters are described in TABLE II. For each elevation column of the 2D feed, the LCMV beamformer is applied over the azimuth channels to ensure a $3 \mathrm{~dB}$ beamwidth of $2.0^{\circ}$ (cf. Fig.14).

The PRI variation follows the considerations in Section II.A: a very long sequence of $N_{P R I}=12197$ pulses repeats cyclically with period $T_{\text {cycle }}=3.52 \mathrm{~s}$ to implement a PRF variation in the range 3400-3534 $\mathrm{Hz}$ (circa 4\%). Meanwhile, 6 simultaneous elevation beams implement multi-SCORE over the swath.

The data rate estimations assume 4-bit block-adaptive quantization (BAQ) and oversampling rates of $26.5 \%$ (with respect to the pulse bandwidth) in elevation, accounting for data headers and guard intervals. In the "raw" case, the azimuth sampling rate is the PRF and in the "minimum" case, which excludes multilooking, an oversampling of $20 \%$ with respect to the processed bandwidth is assumed.

The SAR performance is summarized in Fig.19 in terms of the AASR (a), RASR (b), NESZ (c) (cf. [39] for a discussion of the estimation of this parameter from the patterns and system properties) and azimuth resolution (d).

TABLE II

L-BAND SINGLE-POLARIZATION SLOWLY VARYING PRI MODE PARAMETERS (SINGLE-CHANNEL)

\begin{tabular}{|c|c|c|}
\hline \multicolumn{3}{|c|}{ Pulse and $T x / R x$ hardware parameters } \\
\hline Parameter & Symbol & Value \\
\hline Cycle time & $T_{\text {cycle }}$ & $3.52 \mathrm{~s}$ \\
\hline Gap duration & $T_{g a p}$ & $0.19-0.24 \mathrm{~s}$ \\
\hline Worst-case illumination time & $T_{i l l_{\min }}$ & $3.28 \mathrm{~s}$ \\
\hline PRF range & PRF & $3400-3534 \mathrm{~Hz}$ \\
\hline Total PRI variation & $\triangle P R I$ & $11.18 \mu \mathrm{s}$ \\
\hline Relevant orders & $\left(k_{\min }, k_{\max }\right)$ & $(18,25)$ \\
\hline PRI sequence length & $N_{P R I}$ & 12197 \\
\hline Pulse length (fixed) & $\tau_{p}$ & $14.7 \mu \mathrm{s}$ \\
\hline Minimum duty cycle & $d c_{\min }$ & $5 \%$ \\
\hline Worst-case Doppler centroid & $f_{d c}$ & $892 \mathrm{~Hz}$ \\
\hline Average transmitted power & $P_{T x}$ & $1962 \mathrm{~W}$ \\
\hline Polarization & $-{ }_{-}$ & VV \\
\hline Raw data rate (at PRF) & $R_{\text {raw }}$ & $4215 \mathrm{Mbps}^{6}$ \\
\hline $\begin{array}{l}\text { Minimum data rate } \\
\text { (120\% of processed bandwidth) }\end{array}$ & $R_{\min }$ & $1940 \mathrm{Mbps}$ \\
\hline
\end{tabular}

Processing and beamforming parameters

${ }^{6} 1 \mathrm{Mbps}=2^{20} \mathrm{bps}$ (bits per second)

\begin{tabular}{lll}
\hline Parameter & Symbol & Value \\
\hline Goal azimuth resolution & $\delta_{a z}$ & $5 \mathrm{~m}$ \\
$\begin{array}{l}\text { Processed bandwidth } \\
\text { Number of (simultaneous) } \\
\text { elevation beams }\end{array}$ & $B w_{\text {proc }}$ & $1330 \mathrm{~Hz}$ \\
$\begin{array}{l}\text { Number of active elevation } \\
\text { channels per SCORE beam }\end{array}$ & $N_{\text {beams }}$ & 6 \\
$\begin{array}{l}\text { Sidelobe constraint for SCORE } \\
\text { beamforming }\end{array}$ & $20 \cdot \log _{10}\left(\epsilon_{S D L}\right)^{7}$ & $-36 \mathrm{~dB}$ \\
\hline \hline
\end{tabular}

The impulse responses for the best (e) and worst cases (f), are shown for targets at the center of the swath. These are the references for estimation of the AASR by the Integrated Sidelobe (ISLR) method [28].

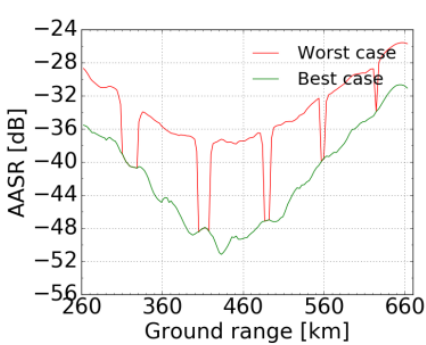

(a)

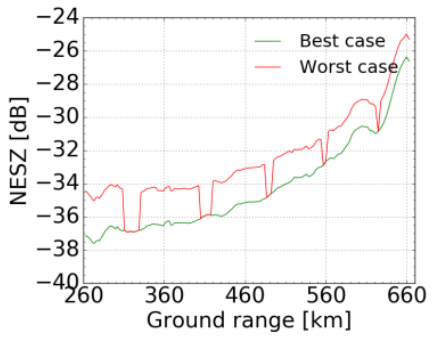

(c)

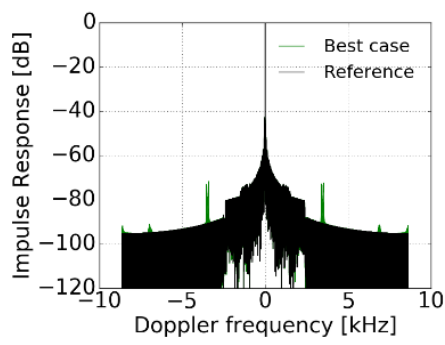

(e)

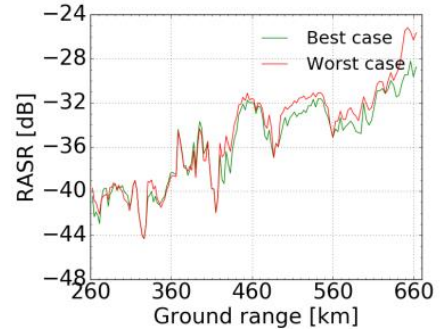

(b)

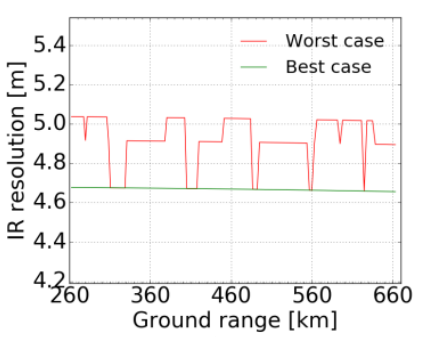

(d)

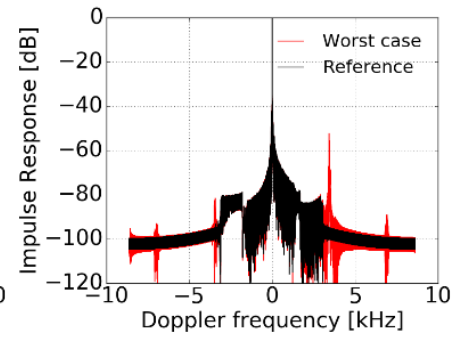

(f)
Fig.19: Simulated SAR performance of reflector system in single-pol singleazimuth channel slowly varying PRI mode. (a) AASR; (b) RASR; (c) NESZ and (d) azimuth resolution. (e) shows the point target impulse response for the center of the swath, for the best case and (f) for the worst case, at the same position. The reference in each case is simulated with an equivalent Doppler spectrum but at a uniform grid and devoid of aliasing, in order to apply the Integrated Sidelobe Ratio (ISLR) based method of [28] for AASR estimation.

For each parameter, the best and worst cases (cf. Section II.B) are represented by green and red curves, respectively. The performance of the high squint case is, as expected, systematically

\footnotetext{
${ }^{7}$ The notation is to emphasize that $-36 \mathrm{~dB}$ is a power constraint: $\epsilon_{S D L}$ denotes the value of the pattern (proportional to electric field) at the sidelobe region.
} 
worst. The AASR and RASR are better than $-25.6 \mathrm{~dB}$ and $-25.2 \mathrm{~dB}$, respectively. The average power of $1962 \mathrm{~W}$ is designed to yield a NESZ better than $-25 \mathrm{~dB}$ in the worst case ${ }^{8}$. The azimuth resolution is better than $4.6 \mathrm{~m}$ in the best case (no squint), and better than 5.1 $\mathrm{m}$ in the worst (highest squint), with local deviations according to the secondary gap position (cf. Section II.B).

The RASR is kept low in spite of the relatively high PRF by usage of the sidelobe-constrained beamformer described in [18], [40]. The SCORE beams are designed to yield a sidelobe level at least $36 \mathrm{~dB}$ below the peak level in the regions which yield range ambiguities, for the whole PRF range. Thus, a broad minimum is formed, as illustrated in Fig.20.

The relatively high first sidelobe levels are caused by the fact that the first-order range ambiguities are close to the main beam, as a consequence of the adopted PRF. This implies a loss in gain and NESZ, which is traded-off for the ambiguity performance.

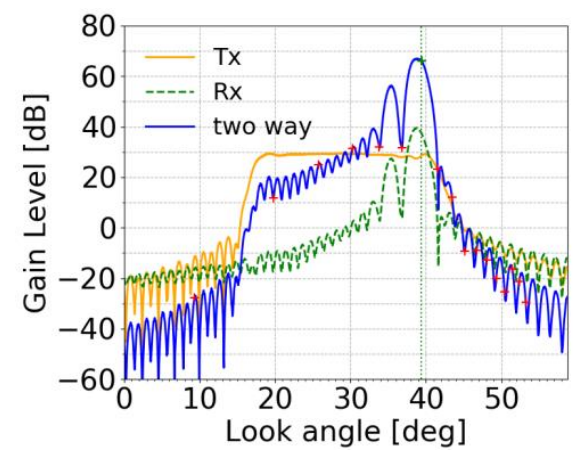

(a)

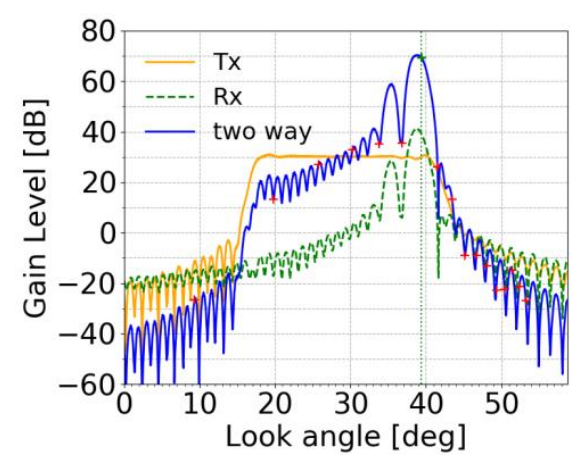

(b)

Fig.20: Elevation patterns in far-range. Tx, Rx and two-way patterns are shown. The signal (green) and ambiguities (red) positions for the mean PRF are denoted by crosses. The Tx pattern (orange) is optimized with phase-only weights for maximum flatness within the main beam, without control over the sidelobe region (this results in the relatively high lobes in near range, which could be compensated at the expense of less flatness using the same algorithm used in Rx). The sidelobe constraint over the Rx pattern is designed to suppress ambiguities for all the PRF range, yielding broad minima. (a) Elevation pattern in the worst case, for which the weights are designed. (b) Elevation pattern of the best case, using the same weights: the gain difference is $\sim 3 \mathrm{~dB}$.

\footnotetext{
${ }^{8}$ The required average power level is rather high for a reflector system, driven by the far-range sensitivity performance. As apparent from the NESZ curve in | Fig.19Fig.19 (c), it could be reduced considerably if the swath extension was relaxed.
}

The RASR values are estimated for the mean PRF of $3467.15 \mathrm{~Hz}$ and the antenna pattern manifold used for the optimization corresponds to the worst-case (with squint). A difference is to be expected due to the non-separability of the reflector patterns. This approach degrades the best-case performance slightly but improves the worst-case performance, which is the driver in terms of the requirements.

\section{B. Single-Pol Slowly Varying PRI Mode: Multichannel in Azimuth}

Next, the same system is used to apply the azimuth beamforming method described in Section III.A. Doppleradaptive beamforming with 16 sub-bands (cf. pattern in Fig.14) is applied, making use of the three azimuth channels. The mode's parameters are described in TABLE III. In this case, the improved azimuth beamforming allows the use of a lower mean PRF, and the timing changes accordingly. The processing and beamforming parameters are the same as in TABLE II.

TABLE III

L-BAND SingLE-POL SLOWLY VARYING PRI MODE PARAMETERS (MULTICHANNEL)

\begin{tabular}{|c|c|c|}
\hline \multicolumn{3}{|c|}{ Pulse and $\mathrm{Tx} / \mathrm{Rx}$ hardware parameters } \\
\hline Parameter & Symbol & Value \\
\hline Cycle time & $T_{\text {cycle }}$ & $3.52 \mathrm{~s}$ \\
\hline Gap duration & $T_{\text {gap }}$ & $0.19-0.24 \mathrm{~s}$ \\
\hline Worst-case illumination time & $T_{i l l_{\min }}$ & $3.28 \mathrm{~s}$ \\
\hline PRF range & PRF & $2850-2985 \mathrm{~Hz}$ \\
\hline Total PRI variation & $\triangle P R I$ & $15.88 \mu \mathrm{s}$ \\
\hline Relevant orders & $\left(k_{\min }, k_{\max }\right)$ & $(15,22)$ \\
\hline PRI sequence length & $N_{P R I}$ & 10262 \\
\hline Pulse length & $\tau_{p}$ & $17.54 \mu \mathrm{s}$ \\
\hline Duty cycle & $d c_{\min }$ & $5 \%$ \\
\hline Worst-case Doppler centroid & $f_{d c}$ & $893 \mathrm{~Hz}$ \\
\hline Average transmitted power & $P_{T x}$ & $617 \mathrm{~W}$ \\
\hline Polarization & - & VV \\
\hline $\begin{array}{l}\text { Raw data rate (at PRF, sum of } \\
\text { all channels) }\end{array}$ & $D_{\text {raw }}$ & $3553 \mathrm{Mbps}$ \\
\hline $\begin{array}{l}\text { Minimum data rate } \\
\text { (processed bandwidth) }\end{array}$ & $D_{\min }$ & $1944 \mathrm{Mbps}$ \\
\hline
\end{tabular}

The SAR performance for this mode is summarized in Fig. 21. The AASR and RASR are better than $-30.4 \mathrm{~dB}$ and $-29.2 \mathrm{~dB}$, respectively, leading to an ASR lower than -27.9 $\mathrm{dB}$. The very low azimuth ambiguity levels are a consequence of the very good sidelobe behavior of the azimuth patterns (cf. Fig.14), in spite of the reduced mean PRF with respect to the previous case.

The required average power to achieve $-25 \mathrm{~dB}$ NESZ is reduced to $617 \mathrm{~W}$, an improvement of circa $5 \mathrm{~dB}$. It should be recognized that part of the improvement comes from the reduced PRF: due to the sidelobe-constrained beamformer, a lower PRF means looser constraints in the vicinity of the main beam (cf. Fig.20), imposing a smaller gain loss. This indicates that the proposed beamforming also brings considerable benefits in terms of the pattern gain. The azimuth resolution is better than $5.0 \mathrm{~m}$. 


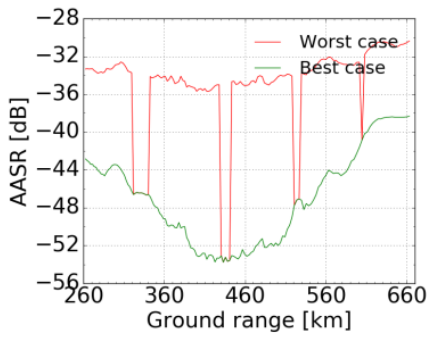

(a)

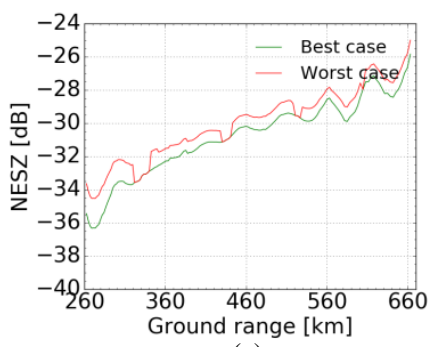

(c)

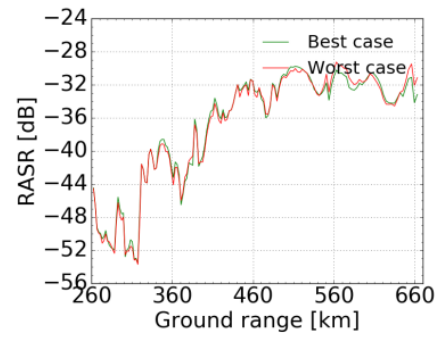

(b)

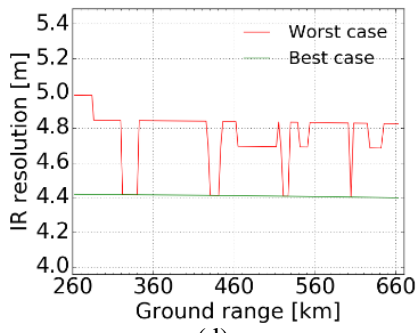

(d)
Fig. 21: Simulated SAR performance of reflector system in single-pol multichannel slowly varying PRI mode. (a) AASR; (b) RASR; (c) NESZ and (d) azimuth resolution.

\section{Quad-Pol Slowly Varying PRI Mode: Single-channel in Azimuth}

In this section, focus is turned to performance in quad-pol. Since the interleaving of $\mathrm{H}$ and $\mathrm{V}$ pulses means it is more challenging to achieve the intended performance levels, the imaging requirements are relaxed. A slow PRI variation mode is designed to cover a 350 $\mathrm{km}$ swath, starting from the same minimum incidence angle as in the previous section. The goal azimuth resolution is now set to 10 $\mathrm{m}$, and the azimuth beamforming is adapted to yield a narrower pattern than in IV.A and IV.B. To achieve a comparable range resolution, the chirp beamwidth is halved to $42 \mathrm{MHz}$. The mode's parameters are summarized in TABLE IV, whereas the SAR performance is summarized in Fig. 22.

TABLE IV

L-BAND QUAD-POLARIZATION SLOWLY VARYING PRI MODE PARAMETERS (SINGLE-CHANNEL)

\begin{tabular}{|c|c|c|}
\hline \multicolumn{3}{|c|}{ Platform and swath parameters } \\
\hline Parameter & Symbol & Value \\
\hline Swath width on ground & $W_{\text {ground }}$ & $350 \mathrm{~km}$ \\
\hline $\begin{array}{l}\text { Swath minimum/maximum } \\
\text { incidence angle }\end{array}$ & $\phi_{\min } / \phi_{\max }$ & $21.0-42.8 \mathrm{deg}$ \\
\hline \multicolumn{3}{|c|}{ Pulse and Tx/Rx Hardware parameters } \\
\hline Parameter & Symbol & Value \\
\hline Cycle time & $T_{\text {cycle }}$ & $1.60 \mathrm{~s}$ \\
\hline Gap duration & $T_{\text {gap }}$ & $0.21-0.25 \mathrm{~s}$ \\
\hline Worst-case illumination time & $T_{i l l}$ & $3.28 \mathrm{~s}$ \\
\hline PRF range & PRF & $3800-3931 \mathrm{~Hz}$ \\
\hline Total PRI variation & $\triangle P R I$ & $8.77 \mu \mathrm{s}$ \\
\hline Relevant orders & $\left(k_{\min }, k_{\max }\right)$ & $(20,27)$ \\
\hline PRI sequence length & $N_{P R I}$ & 7001 \\
\hline Pulse length (fixed) & $\tau_{p}$ & $26.32 \mu \mathrm{s}$ \\
\hline Minimum duty cycle & $d c_{\min }$ & $10 \%$ \\
\hline Average transmitted power & $P_{T x}$ & $880 \mathrm{~W}$ \\
\hline Polarization & - & $\mathrm{HH}, \mathrm{VV}, \mathrm{HV}, \mathrm{VH}$ \\
\hline Raw data rate (at PRF) & $D_{\text {raw }}$ & $3995 \mathrm{Mbps}$ \\
\hline $\begin{array}{l}\text { Minimum data rate } \\
\text { (processed bandwidth) }\end{array}$ & $D_{\min }$ & $1650 \mathrm{Mbps}$ \\
\hline Chirp Bandwidth & $B w_{\text {chirp }}$ & $42 \mathrm{MHz}$ \\
\hline
\end{tabular}

\begin{tabular}{lll}
\hline \hline & Processing and beamforming parameters \\
\hline Parameter & Symbol & Value \\
\hline Goal azimuth resolution & $\delta_{a z}$ & $10 \mathrm{~m}$ \\
$\begin{array}{l}\text { Processed bandwidth } \\
\text { Number of (simultaneous) } \\
\text { elevation beams }\end{array}$ & $B w_{\text {proc }}$ & $665 \mathrm{~Hz}$ \\
$\begin{array}{l}\text { Number of active elevation } \\
\text { channels per SCORE beam }\end{array}$ & $N_{\text {beams }}$ & 6 \\
$\begin{array}{l}\text { Sidelobe constraint for SCORE } \\
\text { beamforming }\end{array}$ & $N_{S C O R E}$ & 11 \\
\hline \hline
\end{tabular}

The AASR (a) levels are better than $-30.3 \mathrm{~dB}$. Note that, due to the interleave, the sampling depends on the Tx polarization, and thus four curves are plotted, including the best and worst case in each Tx polarization. The difference between polarizations (for the same case) is, however, minor; owing to the very similar PRIs for both cases. The RASR levels are shown separately for each case in plots (c) and (d), each of which containing four curves corresponding to the polarizations. The levels of the cross-polarized channels are as expected the limiting factors, in this case below $-24.5 \mathrm{~dB}$. The corresponding ASR is lower than $-24.4 \mathrm{~dB}$.

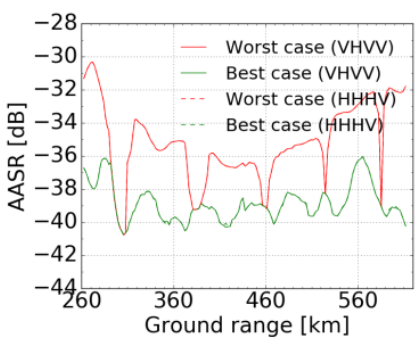

(a)

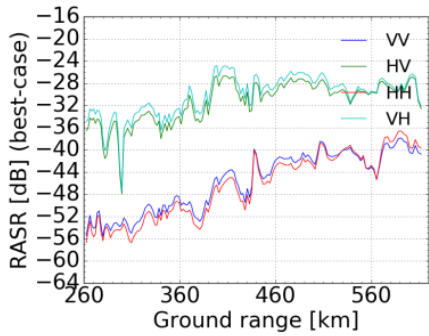

(c)

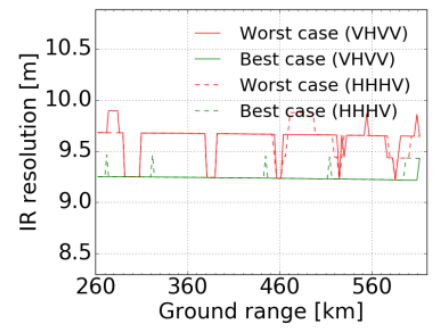

(e)

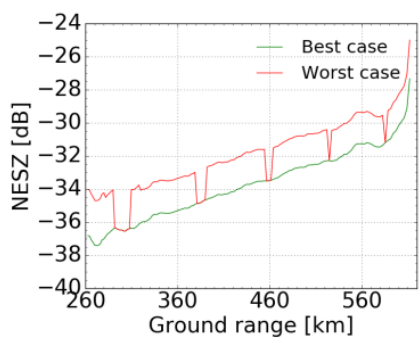

(b)

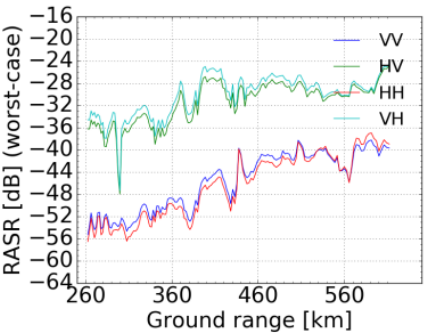

(d)
Fig.22: Simulated SAR performance of reflector system in quad-pol (singlechannel) slowly varying PRI mode. (a) AASR; (b) NESZ; (c) RASR for the best-case (minimum Doppler centroid); (d) RASR for the worst-case (maximum Doppler centroid) and (e) azimuth resolution.

The required average power for $-25 \mathrm{~dB}$ NESZ (b) is in this case $880 \mathrm{~W}$, and the azimuth resolution (e) is better than $9.9 \mathrm{~m}$. Note that the pulse bandwidth is reduced to $42 \mathrm{MHz}$, which 
alongside the reduced swath dimensions accounts for the substantial reduction in the required average power.

\section{Quad-Pol Single-channel Staggered SAR Mode}

As mentioned in the introduction, staggered SAR [16] also represents a HRWS imaging concept in which the PRI is continuously and cyclically changed. The strategy is, however, different, in that a fast PRI variation is employed, with the goal of achieving narrow gaps which can be later compensated by interpolation. In consequence, the spectral gaps of the slowly varying PRI mode are not present. The targets show the same azimuth spectrum regardless of their position, as in a conventional Stripmap. This means that a $5.0 \mathrm{~m}$ resolution can be achieved with the same azimuth patterns as in IV.C. The swath needs however to be reduced to half $(200 \mathrm{~km})$ to achieve comparable range-ambiguity levels.

The mode's parameters are summarized in TABLE V.

TABLE V

L-BAND Single-Channel StagGered Mode Simulation Parameters

Platform and swath parameters

\begin{tabular}{|c|c|c|}
\hline Parameter & Symbol & Value \\
\hline Swath width on ground & $W_{\text {ground }}$ & $200 \mathrm{~km}$ \\
\hline $\begin{array}{l}\text { Swath minimum/maximum } \\
\text { incidence angle }\end{array}$ & $\phi_{\min } / \phi_{\max }$ & $21.0-34.5 \mathrm{deg}$ \\
\hline \multicolumn{3}{|c|}{ Pulse and $\mathrm{Tx} / \mathrm{Rx}$ Hardware parameters } \\
\hline Parameter & Symbol & Value \\
\hline Average PRF & $\overline{\overline{P R F}}$ & $2 \times 3150 \mathrm{~Hz}$ \\
\hline PRF range & PRF & $5924-7800 \mathrm{~Hz}$ \\
\hline Initial PRI & $P R I_{0}$ & $170 \mu \mathrm{s}$ \\
\hline PRI sequence step & $\Delta$ & $-0.28 \mu \mathrm{s}$ \\
\hline PRI sequence length & $N_{P R I}$ & 146 \\
\hline Pulse length (fixed) & $\tau_{p}$ & $9.52 \mu \mathrm{s}$ \\
\hline Average duty cycle & $d c_{\text {mean }}$ & $10 \%$ \\
\hline Average transmitted power & $P_{T x}$ & $81 \mathrm{~W}$ \\
\hline Polarization & - & $\mathrm{HH}, \mathrm{VV}, \mathrm{HV}, \mathrm{VH}$ \\
\hline Raw data rate (at PRF) & $D_{\text {raw }}$ & $6478 \mathrm{Mbps}$ \\
\hline $\begin{array}{l}\text { Minimum data rate } \\
\text { (processed bandwidth) }\end{array}$ & $D_{\min }$ & $3350 \mathrm{Mbps}$ \\
\hline
\end{tabular}

Processing and beamforming parameters

\begin{tabular}{lll}
\hline Parameter & Symbol & Value \\
\hline $\begin{array}{l}\text { Goal azimuth resolution } \\
\text { Processed bandwidth }\end{array}$ & $\delta_{a z}$ & $5 \mathrm{~m}$ \\
$\begin{array}{l}\text { Number of (simultaneous) } \\
\text { elevation beams }\end{array}$ & $N_{\text {beams }}$ & $1360 \mathrm{~Hz}$ \\
$\begin{array}{l}\text { Number of active elevation } \\
\text { channels per SCORE beam }\end{array}$ & $N_{S C O R E}$ & 11 \\
$\begin{array}{l}\text { Sidelobe constraint for SCORE } \\
\text { beamforming }\end{array}$ & $20 \cdot \log _{10}\left(\epsilon_{S D L}\right)$ & $\begin{array}{l}-40 \mathrm{~dB} \text { to }-33 \mathrm{~dB} \\
\text { (curve with } \\
\text { smaller values in } \\
\text { far range) }\end{array}$ \\
\hline \hline
\end{tabular}

The SAR performance is shown in Fig.23. The ambiguity level estimation for staggered SAR follows special considerations described in [28] in detail. The AASR and RASR are better than $-27.4 \mathrm{~dB}$ and $-27.1 \mathrm{~dB}$, respectively, leading to an ASR better than $-25.9 \mathrm{~dB}$. The peaks in the AASR levels are caused by the local sampling configuration, which may lead to larger gaps at specific locations ${ }^{9}$. The required average power for $-25 \mathrm{~dB}$ NESZ is $81 \mathrm{~W}$, and the azimuth resolution is better than $4.8 \mathrm{~m}$.

Given that the same azimuth weights are used as in Section IV.C, both the staggered SAR mode with $5 \mathrm{~m}$ resolution and $200 \mathrm{~km}$ coverage and the slow PRI variation mode with $10 \mathrm{~m}$ resolution and $350 \mathrm{~km}$ coverage could be implemented in the same singlechannel instrument. Another interesting opportunity is to combine a $350 / 400 \mathrm{~km}$ swath mode in Staggered SAR (with the same $5 \mathrm{~m}$ azimuth resolution) in single-pol with the slowly varying PRI mode (with a coarser $10 \mathrm{~m}$ azimuth resolution) as in IV.CIV.C in quadpol, covering in the same swath. This trades-off azimuth resolution for coverage and allows a complementary solution to the strategy of swath reduction highlighted in this section. This strategy was considered as an alternative for ESA's ROSE-L mission proposal [41].

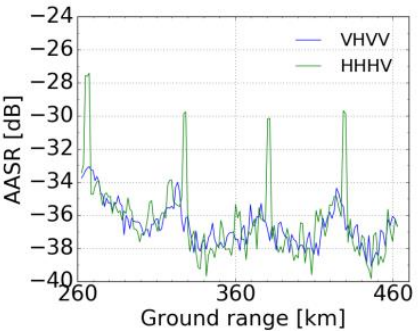

(a)

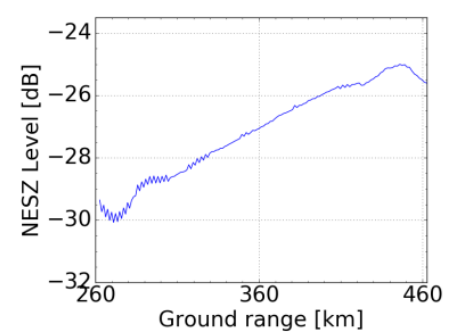

(c)

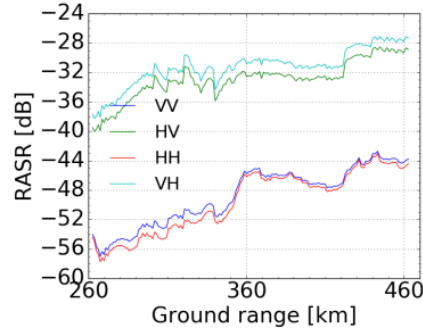

(b)

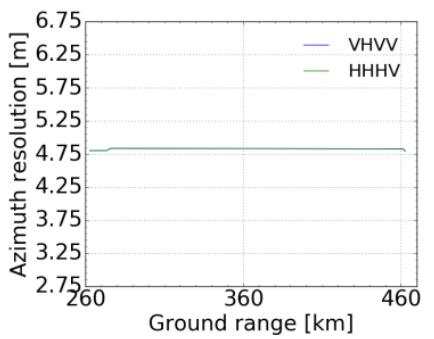

(d)
Fig.23: Simulated SAR performance of reflector system in quad-pol singlechannel staggered SAR mode. (a) AASR; (b) RASR; (c) NESZ ${ }^{10}$ and (d) azimuth resolution.

\section{E. Complementary Multichannel Staggered SAR Mode}

Assuming the system of TABLE I possesses multiple azimuth channels (cf. Section IV.B) and the capability of continuous PRI variation, it may also be employed in a multichannel staggered SAR mode, [17], [18], [42]. In this case, a complementary mode designed to cover the first $200 \mathrm{~km}$ of the single-pol swath with an improved azimuth resolution better than $2.5 \mathrm{~m}$ is considered. The parameters which differ from Section IV.A are listed in TABLE VI, whereas the SAR performance over the swath is depicted in Fig.24. Details of the azimuth beamforming for this case can be found in [17].

\footnotetext{
9 The use of composite PRI sequences [16] is a possible strategy to mitigate this effect, if necessary.

${ }^{10}$ The improvement of the NESZ at very far range is caused by the relaxation of the sidelobe constraint.
} 
TABLE VI

L-BAnd Multichannel StagGered SAR Mode Simulation Parameters

Platform and swath parameters

\begin{tabular}{lll}
\hline Parameter & Symbol & Value \\
\hline Swath width on ground & $W_{\text {ground }}$ & $200 \mathrm{~km}$ \\
$\begin{array}{l}\text { Swath minimum/maximum } \\
\text { incidence angle }\end{array}$ & $\phi_{\min } / \phi_{\max }$ & $24.0-37.0 \mathrm{deg}$ \\
\hline \hline
\end{tabular}

Pulse and Tx/Rx hardware parameters

\begin{tabular}{lll}
\hline Parameter & Symbol & Value \\
\hline Average PRF & $\overline{P R F}$ & $2 \times 2642.5 \mathrm{~Hz}$ \\
Initial PRI & $P R I_{0}$ & $210 \mu \mathrm{s}$ \\
PRI sequence step & $\Delta$ & $-0.62 \mu \mathrm{s}$ \\
PRI sequence length & $N_{P R I}$ & $2 \times 62$ \\
Pulse length & $\tau_{p}$ & $18.92 \mu \mathrm{s}$ \\
Average duty cycle & $d c_{\text {mean }}$ & $10 \%$ \\
Average transmitted power & $P_{T x}$ & $350 \mathrm{~W}$ \\
Polarization & - & $\mathrm{HH} / \mathrm{HV} / \mathrm{VH} / \mathrm{VV}$ \\
Raw data rate (at PRF) & $R_{\text {raw }}$ & $20783 \mathrm{Mbps}$ \\
Minimum data rate & $R_{\min }$ & $6928 \mathrm{Mbps}$ \\
(processed bandwidth) & & \\
\hline \hline
\end{tabular}

Processing and beamforming parameters

\begin{tabular}{lll}
\hline Parameter & Symbol & Value \\
\hline $\begin{array}{l}\text { Goal azimuth resolution } \\
\begin{array}{l}\text { Processed bandwidth } \\
\text { Number of (simultaneous) } \\
\text { elevation beams }\end{array}\end{array} \delta_{a z}$ & $B w_{\text {proc }}$ & $2.5 \mathrm{~m}$ \\
$\begin{array}{l}\text { Number of active elevation } \\
\text { channels per SCORE beam }\end{array}$ & $N_{\text {beams }}$ & $2986.4 \mathrm{~Hz}$ \\
$\begin{array}{l}\text { Sidelobe constraint for SCORE } \\
\text { beamforming }\end{array}$ & $20 \cdot \log _{10}\left(\epsilon_{S D L}\right)$ & $\begin{array}{l}-40 \mathrm{~dB} \text { (relaxed } \\
\text { final } 10 \% \text { of the } \\
\text { swath) }\end{array}$ \\
$\begin{array}{l}\text { Number of samples for azimuth } \\
\text { beamforming }\end{array}$ & $N_{\text {win }}$ & 90 \\
$\begin{array}{l}\text { SNR emphasis parameter of } \\
\text { azimuth beamformer }\end{array}$ & $\alpha$ & 0.0 \\
\hline \hline
\end{tabular}

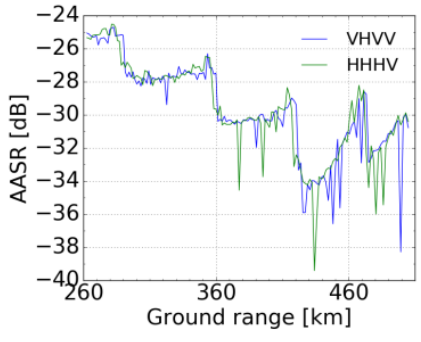

(a)

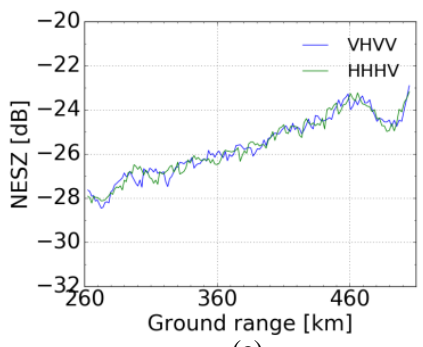

(c)

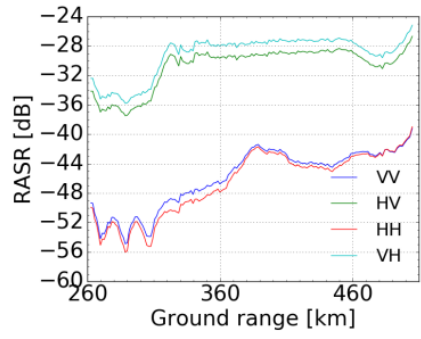

(b)

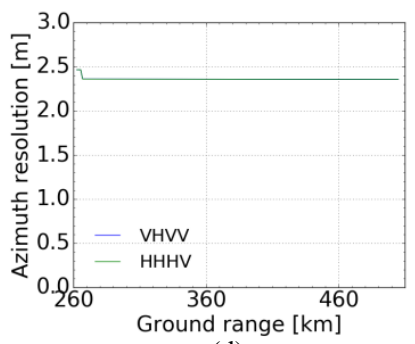

(d)
Fig.24: Simulated SAR performance of reflector system in multichannel staggered SAR mode using Virtual Beam Synthesis. (a) AASR; (b) RASR; (c) NESZ and (d) azimuth resolution.
The AASR and RASR are better than $-24.5 \mathrm{~dB}$ and $-25.2 \mathrm{~dB}$, respectively. The corresponding ASR is better than $-24.0 \mathrm{~dB}$. The -25 $\mathrm{dB} N E S Z$ requirement is achieved with an average power of $350 \mathrm{~W}$, and the resolution is better than $2.5 \mathrm{~m}$. This high-resolution quadpolarization mode is considered an interesting complement to the multichannel slow PRI variation case seen before.

\section{CONCLUSION}

The paper introduced a design criterion for a slowly varying PRI mode (first proposed in [7]) in which a slow, linear and cyclical variation is used to smoothly shift the gaps due to Tx events over the swath. The mode assumes multiple elevation beams and has the peculiarity that the Tx-induced gaps are not recovered as in staggered SAR, but rather lead to gaps in the Doppler spectrum. These impose a Doppler centroid which depends on the target position in along-track and a certain degree of scalloping, as in a ScanSAR. Variations of the mode and a first analysis of the implications for interferometry where also addressed.

Even though reflector antenna architectures possess advantages for the implementation of wide-swath modes [43], in particular for the implementation of (multi-beam) SCORE, the PRI sequence design strategy shown in the paper relies on geometric properties and is applicable to both reflector and planar antennas.

This mode retains some properties of the ScanSAR mode and some of staggered SAR. Note that ScanSAR can be viewed as a particularly simple PRI variation scheme (staircase PRI waveform) and that Staggered SAR involves a more sophisticated fast-PRI variation. Therefore, the slow PRI variation can be understood as an intermediate step between these two, in a trade-off between the speed/complexity of the PRI variation and the effectiveness of the azimuth illumination (in terms of the presence of Doppler spectral gaps, and their duration), which has important consequences for the achievable azimuth resolution at reasonable average power levels.

The slow PRI variation mode, as shown, features shorter spectral gaps than a typical ScanSAR mode, whose gap extension is driven up in a wide-swath scenario by the need to use several sub-swathes. This results in the capability of coping with azimuth resolutions which are finer than those a typical single-channel ScanSAR can achieve over a wide swath, at the cost of the more complex PRI scheme. Following the same logic, this mode will typically be outperformed by staggered SAR for the same azimuth resolution. This occurs as the latter shows the important advantage of a more efficient (stripmap-like) azimuth illumination, without spectral gaps. Clearly, the need to increase the azimuth beamwidth, alongside the scalloping of the worst-case, degrades SAR performance in general (especially in azimuth, e.g. AASR for the same resolution). However, the wider spectrum bears the potential of allowing multilooking or observation of a target with angle of arrival diversity. Furthermore, the slow PRI variation is simpler than the fast PRI variation of staggered SAR, leading to simpler processing than the latter (which is especially relevant for on-board implementation). Moreover, the fact that the azimuth aperture, typically the largest for SAR antennas, is shorter (to implement the wider beams) is an advantage in terms of antenna size. The multiple elevation beam architecture requires multiple channels and a higher 
antenna in elevation; however, the overall antenna area is kept relatively small. Note that a staggered SAR mode typically requires a larger antenna to fulfill the same ambiguity requirements: first, a narrower azimuth beam (for finer resolution) implies a larger azimuth aperture, and, second, the need for PRF oversampling tends to drive up the antenna height (in elevation) to compensate for range ambiguities. Note that, even though the slow PRI variation also relies on oversampling in azimuth (which is the price to counter the non-uniformity, due to the PRI variation), the degree of oversampling is smaller than that of staggered SAR. This is true as the level of non-uniformity is lower and no interpolation across the gaps is performed.

These characteristics mean that this new mode could effectively fill a niche for resolutions in-between those of (single-channel) ScanSAR and those of (single-channel) Staggered SAR. It could thus be an interesting lower-complexity option in comparison to, e.g., a multichannel ScanSAR with similar performance requirements, with the added benefit of a compact antenna design. The fact that the same azimuth beams can be used for Staggered SAR and a slow PRI variation mode with double the azimuth resolution presents furthermore an opportunity for multi-modal operation. The use of the slow PRI variation mode for quad-pol acquisitions combined with a single-pol staggered SAR mode (at half of the azimuth resolution) is considered a particularly interesting example [41], allowing coverage of the same swath (and possibly the same 2D resolution) regardless of the polarization. The Staggered SAR mode imposes more stringent requirements on the hardware (e.g. in terms of the PRI variation and on-board processing), meaning a system capable of fast PRI variation is likely to be able to operate in the slow PRI variation mode without additional complexity.

The convenience of a compact antenna design implies the potential downside of low gain. In the paper, usage of a reflector and exploitation of the nearly-constant PRF to implement a Doppler-frequency dependent azimuth beamforming are proposed as possible alternatives to mitigate this effect. Other possibilities include TOPS or the use of a dedicated Tx antenna using a high-power amplifier, such as a travelling wave tube, which can avoid the use of Transmit/Receive-Modules. This could reduce the losses due to switches, relax constraints on peak power and furthermore reduce overall weight, as the additional antenna is anyhow short and no circulators would be required.

In the paper, several simulation scenarios were provided to illustrate these points, using a reflector system in L-band. All modes were subject to stringent imaging requirements. The single-channel slow PRI variation modes included imaging of a $400 \mathrm{~km}$ swath with $5 \mathrm{~m}$ azimuth resolution in single-pol and a $350 \mathrm{~km}$ swath with $10 \mathrm{~m}$ resolution in quad-pol. The same system could be used to image a $200 \mathrm{~km}$ swath with $5 \mathrm{~m}$ resolution in quad-pol, operating in a complementary staggered SAR mode, extendable to $400 \mathrm{~km}$ in single-pol. Showing good inter-operability with staggered SAR, this mode could represent a complementary mode for e.g. the Tandem- $\mathrm{L}$ mission proposal [44]. A multichannel configuration with 3 azimuth receivers was also considered, imaging the same $400 \mathrm{~km}$ swath with $5 \mathrm{~m}$ resolution in single-pol. In this case, the multichannel configuration gives the slow PRI variation mode a considerable ambiguity performance improvement and also reduces the power requirements. The same variation of the system is employed in a multichannel staggered SAR mode to yield a complementary coverage of the first half of the $400 \mathrm{~km}$ swath with $2.5 \mathrm{~m}$ resolution.

\section{ACKNOWLEDGMENTS}

The authors would like to thank the anonymous reviewers for their helpful comments which aided in making this paper more readable and complete.

\section{REFERENCES}

[1] J. Curlander and R. McDonough, Synthetic Aperture Radar: Systems and Signal Processing, New York: Jon Wiley \& Sons, 1991.

[2] K. Tomiyasu, "Conceptual performance of a satellite borne wide swath synthetic aperture radar," IEEE Transactions on Geoscience and Remote Sensing, Vols. GRS-19, no. 8, pp. 108-116, Aug. 1981.

[3] R. K. Moore, J. P. Claassen and Y. H. Lin, "A scanning spaceborne synthetic aperture radar with integrated radiometer," IEEE Transactions on Aerospace and Electronic Systems, Vols. AES-17, no. 8, pp. 410-421, Aug. 1981.

[4] A. Currie and M. A. Brown, "Wide-swath SAR," IEE Proceedings F - Radar and Signal Processing, vol. 139, pp. 122-135, 1992.

[5] G. Callaghan and I. Longstaff, "Wide-swath spaceborne SAR using a quadelement array," IEE Proc. RSN, vol. 146, pp. 159-165, 1999.

[6] M. Suess, B. Grafmueller and R. Zahn, "A novel high resolution, wide swath SAR system," in IEEE 2001 International Geoscience and Remote Sensing Symposium (IGARSS), Sydney, Australia, 2001.

[7] G. Krieger and .. et al., "Advanced Concepts for Ultra-Wide-Swath SAR Imaging," in 7th European Conference on Synthetic Aperture Radar (EUSAR 2008), Friedrichshafen, Germany, 2008.

[8] G. Krieger, N. Gebert and A. Moreira, "Multidimensional waveform encoding: A new digital beamforming technique for synthetic aperture radar remote sensing," IEEE Transactions on Geoscience and Remote Sensing, vol. 46, no. 8, p. 31-46, Aug. 2008.

[9] G. Krieger et al., "SIMO and MIMO System Architectures and Modes for HighResolution Ultra-Wide-Swath SAR Imaging," in 11th European Conference of Synthetic Aperture Radar (EUSAR), Hamburg, 2016.

[10] M. Younis, F. Queiroz de Almeida, P. López-Dekker and G. Krieger, "Techniques and Modes for Multi-Channel SAR Instruments," in 11th European Conference of Synthetic Aperture Radar (EUSAR), Hamburg, 2016.

[11] N. Gebert, G. Krieger and A. Moreira, "Digital Beamforming on Receive: Techniques and Optimization Strategies for High-Resolution Wide-Swath SAR Imaging," IEEE Transactions on Aerospace and Electronic Systems, vol. 45, no. 8, pp. 564-592, Aug. 2009.

[12] N. Gebert, G. Krieger and A. Moreira, "Multi-Channel ScanSAR for HighResolution Ultra-Wide-Swath Imaging," in 7th European Conference of Synthetic Aperture Radar (EUSAR), Friedrichshafen, Germany, 2008.

[13] M. Suess and W. Wiesbeck, "Side-looking synthetic aperture radar system". European Patent EP 1 241487, Aug. 2002.

[14] A. Freeman et al., "SweepSAR: Beam-forming on receive using a reflectorphased array feed combination for spaceborne SAR," in 2009 IEEE Radar Conference, Pasadena, CA, 2009.

[15] M. Younis, C. Fischer and W. Wiesbeck, "Digital beamforming in SAR systems," in IEEE Transactions on Geoscience and Remote Sensing, 2003.

[16] M. Villano, G. Krieger and A. Moreira, "Staggered SAR: High-Resolution Wide-Swath Imaging by Continuous PRI Variation," IEEE Transactions on Geoscience and Remote Sensing, vol. 52, 2014.

[17] F. Queiroz de Almeida, M. Younis, G. Krieger and A. Moreira, "Multichannel 
Staggered SAR Azimuth Processing," IEEE Transactions on Geoscience and Remote Sensing, vol. 56, no. 5, pp. 2772-2788, Aug. 2018.

[18] F. Queiroz de Almeida, T. Rommel, M. Younis, G. Krieger and A. Moreira, "Multichannel Staggered SAR: System Concepts With Reflector and Planar Antennas," IEEE Transactions on Geoscience and Remote Sensing, vol. 55, no. 2, pp. 877-902, Aug. 2019.

[19] M. Younis, F. Queiroz de Almeida, S. Huber, M. Zonno, M. Rodriguez-Cassola, S. Hensley and G. Krieger, "The Cost of Opportunity for Gapless Imaging," in 2019 IEEE International Geoscience and Remote Sensing Symposium (IGARSS), Yokohama, Japan, 2019.

[20] A. M. Guarnieri and C. Prati, "ScanSAR focusing and interferometry," IEEE Transactions on Geoscience and Remote Sensing, vol. 34, no. 8, pp. 1029-1038, Aug. 1996.

[21] N. Gebert, G. Krieger and A. Moreira, "Multichannel Azimuth Processing in ScanSAR and TOPS Mode Operation," IEEE Transactions on Geoscience and Remote Sensing, vol. 48, no. 8, pp. 2994-3008, 2010.

[22] P. Prats, R. Scheiber, J. Mittermayer, A. Meta and A. Moreira, "Processing of Sliding Spotlight and TOPS SAR Data Using Baseband Azimuth Scaling," IEEE Transactions on Geoscience and Remote Sensing, vol. 48, no. 2, pp. 770 780, Feb. 2010.

[23] S. Wollstadt, P. López-Dekker, F. De Zan and M. Younis, "Design Principles and Considerations for Spaceborne ATI SAR-Based Observations of Ocean Surface Velocity Vectors," IEEE Transactions on Geoscience and Remote Sensing, vol. 55, no. 8, pp. 4500-4519, Aug. 2017.

[24] H. L. Van Trees, Optimum Array Processing, New York: John Wiley \& Sons Inc., 2002.

[25] S. Huber, A. Patyuchenko, G. Krieger and A. Moreira, "Spaceborne Reflector SAR Systems with Digital Beamforming," IEEE Transactions on Aerospace and Electronic Systems, vol. 48, no. 8, pp. 3473-3493, 2012.

[26] F. De Zan and A. Monti Guarnieri, "TOPSAR: Terrain Observation by Progressive Scans," IEEE Transactions on Geoscience and Remote Sensing, vol. 44, no. 8, pp. 2352-2360, Aug. 2006

[27] A. Meta, P. Prats, U. Steinbrecher, J. Mittermayer and R. Scheiber, "TerraSARX TOPSAR and ScanSAR comparison," in 7th European Conference on Synthetic Aperture Radar, Friedrichshafen, Germany, 2008.

[28] M. Villano, G. Krieger and A. Moreira, "Ambiguities and image quality in staggered SAR," in 5th Asia-Pacific Conference on Synthetic Aperture Radar (APSAR), Singapore, 2015.

[29] S. Barbarossa, "Detection and imaging of moving objects with synthetic aperture radar. 1. Optimal detection and parameter estimation theory," IEE Proceedings $F$ - Radar and Signal Processing, vol. 139, no. 8, pp. 79-88, Aug. 1992.

[30] S. M. Kay, Fundamentals of Statistical Signal Processing: Estimation Theory, USA: Prentice Hall, 1993.

[31] J. Dall and A. Kusk, "Azimuth phase coding for range ambiguity suppression in SAR," in 2004 IEEE International Geoscience and Remote Sensing Symposium, Anchorage, AK, 2004.

[32] M. Villano, G. Krieger and A. Moreira, "New Insights Into Ambiguities in Quad-Pol SAR," IEEE Transactions on Geoscience and Remote Sensing, vol. 55, no. 6, pp. 3287-3308, July 2017.

[33] A. Meta, J. Mittermayer, P. Prats, R. Scheiber and U. Steinbrecher, "TOPS Imaging With TerraSAR-X: Mode Design and Performance Analysis," IEEE Transactions on Geoscience and Remote Sensing, vol. 48, no. 2, pp. 759-769, Aug. 2010.

[34] N. Yagüe-Martínez, P. Prats-Iraola, F. Rodríguez González, R. Brcic, R. Shau, D. Geudtner, M. Eineder and R. Bamler, "Interferometric Processing of Sentinel1 TOPS Data," IEEE Transactions on Geoscience and Remote Sensing, vol. 54, no. 4, pp. 2220-2234, Aug. 2016.

[35] P. Prats-Iraola, M. Rodriguez-Cassola, F. De Zan, R. Scheiber, P. López-Dekker, I. Barat and D. Geudtner, "Role of the Orbital Tube in Interferometric Spaceborne SAR Missions," IEEE Geoscience and Remote Sensing Letters, vol. 12, no. 7, pp. 1486-1490, Aug. 2015

[36] P. Prats-Iraola, R. Scheiber, L. Marotti, S. Wollstadt and A. Reigber, "TOPS Interferometry With TerraSAR-X," IEEE Transactions on Geoscience and Remote Sensing, vol. 50, no. 8, pp. 3179-3188, Aug. 2012.

[37] R. Scheiber and A. Moreira, "Coregistration of interferometric SAR images using spectral diversity," IEEE Transactions on Geoscience and Remote Sensing, vol. 38, no. 5, pp. 2179-2191, Aug. 2000.
[38] L. Lei, G. Zhang and R. J. Doviak, "Bias Correction for Polarimetric PhasedArray Radar With Idealized Aperture and Patch Antenna Elements," IEEE Transactions on Geoscience and Remote Sensing, vol. 51, no. 1, pp. 473-486, Aug. 2013.

[39] M. Younis, P. López-Dekker and G. Krieger, "Signal and noise considerations in multi-channel SAR," in 16th International Radar Symposium (IRS), Dresden, Germany, 2015.

[40] S. Huber, F. Queiroz de Almeida, M. Villano, M. Younis, G. Krieger and A Moreira, "Tandem-L: A Technical Perspective on Future Spaceborne SAR Sensors for Earth Observation," IEEE Transactions on Geoscience and Remote Sensing, vol. 56, no. 8, pp. 4792-4807, Aug. 2018.

[41] M. Di Salvo, A. Perrera, M. Degiorgi, A. Ritorto, L. Vinciguerra, F. Temussi, M. Villano, G. Krieger, F. Q. d. Almeida, J. Reimann, M. Zonno and M. Younis, "ROSE-L EO System - Mission and Instrument Performance Assessment," in Proceedings of Workshop on Advanced RF Sensors and Remote Sensing Instruments (ARSI), Noordwijk, The Netherlands, 2019.

[42] F. Queiroz de Almeida and G. Krieger, "Multichannel Staggered SAR Azimuth Sample Regularization," in 11th European Conference on Synthetic Aperture Radar (EUSAR 2016), Hamburg, Germany, 2016.

[43] M. Younis, S. Huber, A. Patyuchenko, F. Bordoni and G. Krieger, "Performance Comparison of Reflector- and Planar-Antenna Based Digital Beam-Forming SAR," International Journal of Antennas and Propagation, 2009.

[44] A. Moreira, G. Krieger, I. Hajnsek, K. Papathanassiou, Y. M., L.-D. P., S. Huber, M. Villano, M. Pardini, M. Eineder, F. De Zan and A. Parizzi, "Tandem-L: A Highly Innovative Bistatic SAR Mission for Global Observation of Dynamic Processes on the Earth's Surface," IEEE Geoscience and Remote Sensing Magazine, vol. 3, no. 2, pp. 8-23, June 2015. 